\title{
Toward Space-like Photometric Precision from the Ground with Beam-shaping Diffusers
}

\author{
Gudmundur Stefansson ${ }^{1,2,3,14,15}$ (1) , Suvrath Mahadevan ${ }^{1,2,3}$ (1) Leslie Hebb $^{4}$ (1) , John Wisniewski ${ }^{5}$ (1), Joseph Huehnerhoff ${ }^{6,7,8}$,

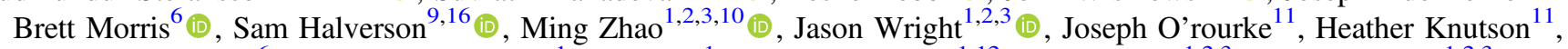 \\ Suzanne Hawley ${ }^{6}\left(\mathbb{0}\right.$, Shubham Kanodia ${ }^{1}$, Yiting $\mathrm{Li}^{1}$, Lea M. Z. Hagen ${ }^{1,12}$ (1), Leo J. Liu ${ }^{1,2,3}$, Thomas Beatty ${ }^{1,2,3} \mathbb{C}^{1}$, \\ Chad Bender ${ }^{1,2,13}$ (10), Paul Robertson ${ }^{1,2,3,16}{ }^{(\mathbb{0}}$, Jack Dembicky ${ }^{7}$, Candace Gray ${ }^{7}$, William Ketzeback ${ }^{7}$, Russet McMillan ${ }^{7}$, and \\ Theodore Rudyk ${ }^{7}$ \\ ${ }^{1}$ Department of Astronomy \& Astrophysics, The Pennsylvania State University, 525 Davey Lab, University Park, PA 16802, USA; gudmundur@psu.edu \\ ${ }_{2}$ Center for Exoplanets \& Habitable Worlds, University Park, PA 16802, USA \\ ${ }^{3}$ Penn State Astrobiology Research Center, University Park, PA 16802, USA \\ ${ }^{4}$ Department of Physics, Hobart and William Smith Colleges, 300 Pulteney Street, Geneva, NY, 14456, USA \\ ${ }^{5}$ Homer L. Dodge Department of Physics and Astronomy, University of Oklahoma, 440 W. Brooks Street, Norman, OK 73019, USA \\ ${ }_{7}^{6}$ Department of Astronomy, Box 351580, University of Washington, Seattle, WA 98195, USA \\ 7 Apache Point Observatory, 2001 Apache Point Road, sunspot, New Mexico, NM 88349, USA \\ ${ }^{8}$ Hindsight Imaging, Inc., 233 Harvard Street, Suite 316, Brookline, MA 02446, USA \\ ${ }^{9}$ Department of Physics and Astronomy, University of Pennsylvania, Philadelphia, PA 19104, USA \\ ${ }^{10}$ The New York Times, 620 Eight Avenue, New York, NY 10018, USA \\ ${ }_{11}^{11}$ Division of Geological and Planetary Sciences, California Institute of Technology, Pasadena, CA 91125, USA \\ ${ }^{12}$ Institute for Gravitation and the Cosmos, The Pennsylvania State University, University Park, PA 16802, USA \\ ${ }^{13}$ Steward Observatory, University of Arizona, 933 N. Cherry Avenue, Tucson, AZ 85719, USA \\ Received 2017 April 28; revised 2017 August 22; accepted 2017 August 22; published 2017 October 5
}

\begin{abstract}
We demonstrate a path to hitherto unachievable differential photometric precisions from the ground, both in the optical and near-infrared (NIR), using custom-fabricated beam-shaping diffusers produced using specialized nanofabrication techniques. Such diffusers mold the focal plane image of a star into a broad and stable top-hat shape, minimizing photometric errors due to non-uniform pixel response, atmospheric seeing effects, imperfect guiding, and telescope-induced variable aberrations seen in defocusing. This PSF reshaping significantly increases the achievable dynamic range of our observations, increasing our observing efficiency and thus better averages over scintillation. Diffusers work in both collimated and converging beams. We present diffuser-assisted optical observations demonstrating $62_{-16}^{+26} \mathrm{ppm}$ precision in 30 minute bins on a nearby bright star $16 \mathrm{Cygni} \mathrm{A}(V=5.95)$ using the ARC $3.5 \mathrm{~m}$ telescope-within a factor of $\sim 2$ of Kepler's photometric precision on the same star. We also show a transit of WASP-85-Ab $(V=11.2)$ and TRES-3b $(V=12.4)$, where the residuals bin down to $180_{-41}^{+66} \mathrm{ppm}$ in 30 minute bins for WASP-85-Ab-a factor of $\sim 4$ of the precision achieved by the $K 2$ mission on this targetand to $101 \mathrm{ppm}$ for TRES-3b. In the NIR, where diffusers may provide even more significant improvements over the current state of the art, our preliminary tests demonstrated $137_{-36}^{+64} \mathrm{ppm}$ precision for a $K_{S}=10.8$ star on the 200 inch Hale Telescope. These photometric precisions match or surpass the expected photometric precisions of TESS for the same magnitude range. This technology is inexpensive, scalable, easily adaptable, and can have an important and immediate impact on the observations of transits and secondary eclipses of exoplanets.
\end{abstract}

Key words: instrumentation: miscellaneous - planets and satellites: fundamental parameters - techniques: photometric

Supporting material: animations, data behind figures

\section{Introduction}

Exoplanet science has seen an explosion in productivity over the past decade. The Kepler spacecraft (Borucki et al. 2010) has detected over 3000 planet candidates (Burke et al. 2015). However, many of the Kepler stars are faint and difficult to follow up with ground-based facilities. After the failure of the second Kepler reaction wheel, the repurposed Kepler mission, $K 2$, has sampled a different population of host stars, namely, more nearby and brighter stars, better suited for follow-up efforts from the ground. This has resulted in synergistic efforts from space and the ground to rapidly confirm and verify new planet candidates (e.g., Vanderburg \& Johnson 2014; Crossfield et al. 2015; Vanderburg et al. 2016).

\footnotetext{
${ }_{15}^{14}$ NASA Earth and Space Science Fellow.

15 Leifur Eiriksson Foundation Fellow.

16 NASA Sagan Fellow.
}

The Transiting Exoplanet Survey Satellite (TESS) is scheduled for launch in 2018 (Ricker et al. 2014). TESS will survey the whole sky for transiting exoplanets around the nearest and brightest stars, and is expected to find thousands of Neptunes and dozens of Earth-sized planets (Ricker et al. 2014; Sullivan et al. 2015). However, the majority of TESS targets will only be observed for 26 days, with significantly larger observational coverage only at the north and south ecliptic poles. Therefore, most of the planet candidates will only have a few transits observed and will require timely ground-based follow-up to confirm their planetary nature.

As such, follow-up observations from the ground, both photometric and spectroscopic, will be crucial in maximizing the TESS yield (Plavchan et al. 2015). Follow-up of promising TESS candidates rapidly after discovery will also enable the community to best use valuable James Webb Space Telescope $(J W S T)$ time for precise atmospheric characterization via transit 
spectroscopy (e.g., Cowan et al. 2015; Batalha et al. 2017; Benneke et al. 2017), and determine how best to align efforts to study the full phase curves of exoplanets to characterize the thermal profiles of their atmospheres.

However, on the ground, telescopes have to contend with the deleterious effects of the atmosphere, including scintillationthe observed intensity variations (or "twinkling") of starstransparency variations, differential extinction, seeing, and telescope-guiding effects, all of which limit the achievable photometric precision.

There have been successes in circumventing these problems to achieve high differential photometric precisions from the ground. Although a detailed comparison of diffuser-assisted photometry with current state of the art is presented in Section 7, we briefly discuss some current techniques and the precision levels achieved with them here for context.

To reach high precisions, modern detectors can be read out quickly with low read noise and images co-added to reach high signal strengths (e.g., Kundurthy et al. 2013, achieving $306 \mathrm{ppm}_{\text {minute }}^{-1}$ with a fast frame transfer CCD). Furthermore, narrow-band filters can be used to desensitize photometric measurements from water column density changes, telluric absorption variations, and atmospheric emission line fluctuations, and to observe bright stars with modest and large-size telescopes (e.g., Colón et al. 2012, achieving 455 ppm minute ${ }^{-1}$ with a $10 \mathrm{~m}$ telescope and a narrow-band filter). Narrow-band filters can also be used in conjunction with polarimetry in novel specialized instruments, such as PEPPER (Polarization Encoding differential Photometer and PolarimetER), to reach highprecision self-differential photometry on a single star without any reference stars (Potter 2006). Perhaps the most popular technique to reach high-precision photometry from the ground is to defocus the telescope to spread the light over many pixels, decreasing sensitivity to individual pixel effects, increasing observing efficiency, and allowing more light to be collected per integration. This has been done successfully by many groups (e.g., Southworth et al. 2009; Croll et al. 2011; Mann et al. 2011; Zhao et al. 2014; Fukui et al. 2016), and excellent results have been reported using defocusing with conventional CCDs in the optical (e.g., Southworth et al. 2009 achieved 434 and $385 \mathrm{ppm}^{\text {minute }}{ }^{-1}$ photometric precisions using a $3.58 \mathrm{~m}$ telescope, and Fukui et al. 2016 achieved 423 ppm minute ${ }^{-1}$ photometric precisions on a $1.88 \mathrm{~m}$ telescope), and also using NIR detector arrays (Croll et al. 2011 achieved $860 \mathrm{ppm}$ minute $^{-1}$ using a $3.6 \mathrm{~m}$ telescope, and Zhao et al. 2014 achieved $3195 \mathrm{ppm}$ minute $^{-1}$ using a $5 \mathrm{~m}$ telescope). Although capable of yielding very high-precision photometry, defocusing the telescope can result in location-dependent aberrations in the point-spread function (PSF) and bright spots that vary with seeing (Southworth et al. 2009) that can saturate the detector. Defocusing can also affect guiding precision (which in turn degrades photometric precision) unless the guider has an independent focusing mechanism. Orthogonal-transfer CCDs (e.g., Tonry et al. 1997; Howell et al. 2003; Johnson et al. 2009) can be used to shape the PSF on the detector itself without needing to defocus, which has been shown by Johnson et al. (2009) to demonstrate excellent photometric precisions of $539 \mathrm{ppm}^{\text {minute }}{ }^{-1}$ on a $2.2 \mathrm{~m}$ telescope. Although this may potentially be more robust and repeatable than the defocusing method, this method requires custom orthogonal-transfer CCDs, which are still not very common. We again refer the reader to Section 7, which further discusses these efforts, puts them in further context, and compares them to the precision levels achieved in this work with diffusers.

In this work, we present a new and inexpensive technology to reliably reach high photometric precisions on bright stars, even in suboptimal observing conditions. We use a custom beam-shaping diffuser, created using specialized nanofabrication techniques, to deterministically "mold" the stellar image into a stable top-hat pattern. By using this diffuser, we minimize atmospheric effects without defocusing the telescope. Furthermore, by spreading the light over many pixels, we minimize flat-fielding errors, while simultaneously increasing observing efficiency, allowing us to observe bright stars reliably without saturating. This technology is versatile, offers broadband compatibility, and is capable of stabilizing stellar PSFs with diffusers placed in either converging or collimated beams. While such diffusers have been briefly explored in the context of precision photometry for the upcoming CHaracterising ExOPlanet Satellite (CHEOPS) mission (Magrin et al. 2014), they were not part of the final CHEOPS design. This work represents the first published results of detailed characterization, testing, and on-sky results using diffuser-assisted photometry. Specifically, in the optical, we present on-sky high-precision demonstrations on Penn State's PlaneWave CDK 24 inch telescope of $246_{-81}^{+176} \mathrm{ppm}$ in 30 minute bins. Also in the optical, we present diffuser-assisted observations performed on the Astrophysical Research Consortium (ARC) $3.5 \mathrm{~m}$ Telescope at APO using the Astrophysical Research Consortium Telescope Imaging Camera (ARCTIC; Huehnerhoff et al. 2016) of 16 Cyg A and the transits of WASP-85 A b and TRES-3b, demonstrating precisions of $62_{-16}^{+26} \mathrm{ppm}, 180_{-41}^{+66} \mathrm{ppm}$, and $\sim 101 \mathrm{ppm}$ in 30 minute bins, respectively. Lastly, we present high-precision photometry in the near-infrared (NIR) on the 200 inch Hale telescope at Palomar using the Wide-field Infrared Camera (WIRC) (Wilson et al. 2003), with a precision of $137_{-36}^{+64} \mathrm{ppm}$ in 30 minute bins. Our optical observations on ARCTIC match or surpass the precisions that are expected of the TESS spacecraft (Ricker et al. 2014; Sullivan et al. 2015) for the same stellar magnitude range in the same binning timescale. ${ }^{17}$

The 30 minute diffuser-assisted photometric precision levels presented in this paper are now beginning to approach (and in some cases exceed) $80 \mathrm{ppm}$ - the transit depth of an Earth around a Sun-like star, even in the presence of scintillation noise. We stress that diffusers can be used to improve the precisions across different telescope apertures. However, we expect that the most significant precision gains beyond the precisions we report here will most likely come from incorporating diffusers on the largest telescopes, such as on the upcoming HiPERCAM on the $10 \mathrm{~m}$ Gran Telescopio Canarias (GTC) or the new OCTOCAM instrument (de Ugarte Postigo et al. 2016) for the $8 \mathrm{~m}$ Gemini telescopes, or on telescopes equipped with conjugate plane photometers to correct for scintillation (Osborn et al. 2011).

This paper is structured as follows. Section 2 discusses the issues and mitigation strategies in achieving high-precision photometry from the ground, setting the stage for the utility of diffusers. Section 3 gives a description of diffusers and how

\footnotetext{
17 Although our NIR precision of $137_{-36}^{+64} \mathrm{ppm}$ in 30 minutes on a $K_{S}=10.8$ mag star is also better than the expected precision of TESS on a $I_{C}=10.8 \mathrm{mag}$ star, comparing our WIRC NIR results to TESS is not completely analogous to comparing our optical results on ARCTIC to TESS, as we discuss further in Section 7.1
} 
Table 1

Absolute Values of Variances in Equation (1) along with the Underlying Distribution of the Variables

\begin{tabular}{lll}
\hline \hline Variance & Distribution & Absolute Value \\
\hline$V_{*}$ & Poisson & $\left|G F_{*}\right|$ \\
$V_{S}$ & Poisson & $\left|G F_{S}\right|$ \\
$V_{D}$ & Poisson & $\left|F_{D}\right|$ \\
$V_{R}$ & Gaussian & $\left|F_{R}^{2}\right|$ \\
$V_{f}$ & Uniform & $\left|G^{2} \sigma_{f}^{2}\right|$ \\
\hline
\end{tabular}

Note. As presented in Collins et al. (2017), $F_{*}$ is the net background-subtracted counts in the aperture from the star in ADUs, $F_{S}$ is the sky-background signal in ADU pixel ${ }^{-1}, F_{D}$ is the dark current signal in electrons pixel ${ }^{-1}$, and $F_{R}$ is the read noise in (electrons pixel ${ }^{-1}$ ) $\operatorname{read}^{-1}$, and $\sigma_{f}$ is an estimate of the $1 \sigma$ error introduced within the A/D converter with a value of $\sim 0.289$ ADU (Merline \& Howell 1995; Collins et al. 2017).

they can be used in telescopes for precision photometry applications. In Section 4, we describe our lab test setup and our observations in the optical and the NIR. We present our lab and on-sky results in Section 5. Section 6 discusses our MCMC modeling and fits for the WASP-85 A b and TRES-3b transits. Section 7 provides further discussion and remarks on this technology and its applicability for use on other telescopes. We conclude in Section 8 with a summary of our findings.

\section{Reaching High Photometric Precisions from the Ground}

The empirical differential photometric precision achieved from telescopes in space and on the ground is well-described by the theoretical calculation of noise for a well-behaved CCD (Merline \& Howell 1995). Similar to the formalism outlined in Collins et al. (2017), the total photometric noise $N$ (excluding scintillation) in ADU (analog-to-digital units) for a CCD aperture photometry measurement is

$$
N=\frac{\sqrt{V_{*}+n_{\mathrm{pix}}\left(1+\frac{n_{\mathrm{pix}}}{n_{b}}\right)\left(V_{S}+V_{D}+V_{R}+V_{f}\right)}}{G},
$$

where $G$ is the gain of the CCD in electrons $\mathrm{ADU}^{-1}, V_{*}$ is the variance of the net background-subtracted counts in the aperture from the star (units: electrons ${ }^{2}$ ), $n_{\text {pix }}$ is the number of pixels in the aperture, $n_{b}$ is the number of pixels used to estimate the mean background sky signal, $V_{S}$ is the variance in the sky-background signal per pixel (units: electrons ${ }^{2}$ pixel $^{-1}$ ), $V_{D}$ is the variance in the dark current signal per pixel (units: electrons ${ }^{2}$ pixel $\left.^{-1}\right), V_{R}$ is the variance of the read noise per pixel (units: electrons ${ }^{2}$ pixel $^{-1}$ ), and the last term $V_{f}$ is the variance in the digitization noise within the $\mathrm{A} / \mathrm{D}$ converter (units: electrons $^{2}$ pixel $^{-1}$ ). Table 1 lists the absolute values of the variances in Equation (1), relating them to the corresponding fluxes measured in number of ADUs or electrons as outlined in Merline \& Howell (1995) and Collins et al. (2017).

Using a similar formalism to Collins et al. (2017), the final normalized relative flux error on the relative flux $F_{\text {rel flux }}=F_{T} / F_{E}$ (where $F_{T}$ is the flux from the target star in $\mathrm{ADU}$ and $F_{E}$ is the total integrated flux from the ensemble in
ADU) is calculated using an ensemble of reference stars, using

$$
\sigma_{\text {rel flux }}=F_{\text {rel flux }} \cdot \sqrt{\frac{N_{T}^{2}}{F_{T}^{2}}+\frac{N_{E}^{2}}{F_{E}^{2}}},
$$

where $N_{T}$ and $N_{E}$ are the noise from the target and the ensemble in ADU, respectively. For each individual star, the noise is calculated using Equation (1), and for the stars in the ensemble, the noise $N_{E}$ is the total noise from all of the reference stars added in quadrature. In normalized units, the corresponding relative flux error is given by

$$
\frac{\sigma_{\text {rel flux }}}{F_{\text {rel flux }}}=\sqrt{\frac{N_{T}^{2}}{F_{T}^{2}}+\frac{N_{E}^{2}}{F_{E}^{2}}} .
$$

Hereafter, we will refer to $\sigma_{\text {rel flux }}$ in these normalized units.

It is instructive to study Equations (1) and (3) in the special case, where we assume minimal errors other than photon noise (i.e., near-perfect detector and minimal background sky noise) and a detector gain close to $G \sim 1$. In this case, the total noise in ADU is simply $N \sim \sqrt{F}$. Extending this to observations of a target star with numerous bright nearby reference stars (i.e., where the photon noise contribution from the reference ensemble is minimal: $F_{E} \sim 0$ ), we then see from Equation (3) that

$$
\sigma_{\text {rel flux }}=\sqrt{\frac{N_{T}^{2}}{F_{T}^{2}}} \sim \sqrt{\frac{F_{T}}{F_{T}^{2}}} \sim \frac{1}{\sqrt{F_{T}}} \sim \frac{1}{N_{T}},
$$

i.e., the normalized relative flux error also reduces to a similar square-root dependence on the target flux in ADU.

Photometric errors are also introduced by instrumental effects, which are not included in these equations, such as the inhomogeneous pixel response of detectors and sensitivity to pixel position. Light curves are often decorrelated in some manner with respect to instrumental parameters in order to remove these effects from both space-based and ground-based photometry. Photometric errors are also introduced by the limited electron well depth of detectors limiting integration times before saturation, which is problematic especially for larger telescopes observing bright stars such as the TESS stars.

From the ground, the precision of ground-based telescopes is further limited due to the deleterious effects of the Earth's atmosphere. Transparency fluctuations, sky-background noise, scintillation, and differential extinction can all affect photometric precision. In particular, scintillation due to turbulence in the upper atmosphere is a particularly insidious source of photometric error for ground-based photometry (Young 1967; Dravins et al. 1998; Kornilov 2012; Osborn et al. 2015). Here we discuss the error sources particular to ground-based photometry and our mitigation strategies using diffusers.

\subsection{Atmospheric Noise Sources}

\subsubsection{Transparency Variations}

Transparency variations include shifting cloud cover. The exact variations will depend on the weather and the observing site, and as such, this source of noise is particularly difficult to estimate for any given observation. This is generally minimized through differential photometry, where the core assumption is that the transparency variations affect the target and reference stars equally. Transparency variations are further minimized by performing observations from a good observing site at high 
elevation. Efforts have been made to estimate the impact of this effect for some observing sites (e.g., Mann et al. 2011) using different atmospheric models, demonstrating that the median noise due to transparency variations is typically smaller than Poisson and scintillation noise for photometric nights (Mann et al. 2011). For our observations, we assume that for a clear photometric night at a good observing site, this error source is typically much smaller than the expected Poisson and scintillation noise.

\subsubsection{Molecular Absorption and Differential Extinction}

A related issue to transparency variations is variable molecular absorption. Commonly used broadband filters, such as the SDSS $u^{\prime} g^{\prime} r^{\prime} i^{\prime} z^{\prime}$ (Fukugita et al. 1996) and UBVRI Johnson-Cousins filters (Bessell 1990), each operate over a wide bandpass and include a number of molecular absorption lines (with water, oxygen, and ozone being the primary absorbers in the optical). For water, the depth of these lines is strongly dependent on the water column at the time of observation, which is dependent on the exact weather conditions and the airmass of the target being observed. This effect is minimized by observing in a bandpass not contaminated by such lines.

Differential extinction is of two types: first order and second order. First-order differential extinction is caused by the variation in the airmass difference of the target and reference star throughout the observation, resulting in a relative brightness change. Mann et al. (2011) estimated that the magnitude of this effect at Maunakea can be on the order of $\sim 10^{-4}-10^{-3}$, depending on the observing conditions and passband being used. However, being a systematic trend correlated with airmass, Mann et al. (2011) mention that this effect can generally be detrended out at high precision if the extinction variation is minimal. Second-order differential extinction is caused by the target and the reference star not being of the same spectral type. Stars with different spectral types will vary differently with extinction throughout the observation. This color effect is smaller in redder passbands, with stars of later spectral type and narrower bandwidth filters, and can further be minimized by a judicious choice of reference stars of the same or similar spectral types.

Both molecular absorption and extinction can be minimized by observing in a red-optical bandpass filter with little to no molecular absorption lines (Mann et al. 2011). We explored the parameter space of commercially available filters and converged on an off-the-shelf filter from Semrock (part number: $857 / 30$ ), operating in a red passband between 842 and $872 \mathrm{~nm} .{ }^{18}$ Figure 1 shows the transmission curve of this filter, along with the typical molecular absorption bands calculated by TERRASPEC (Bender et al. 2012) around this region. As this filter is centered at the red end of the optical spectrum, the recorded photometric signal will be less sensitive to variable Rayleigh scattering.

Thus, with an informed choice of bandpass filter in the redoptical, minimally contaminated by molecular absorption lines, we assume scintillation and photon noise to be the dominant error sources.

\footnotetext{
18 https://www.semrock.com/FilterDetails.aspx $? \mathrm{id}=$ =FF01-857/30-25
}

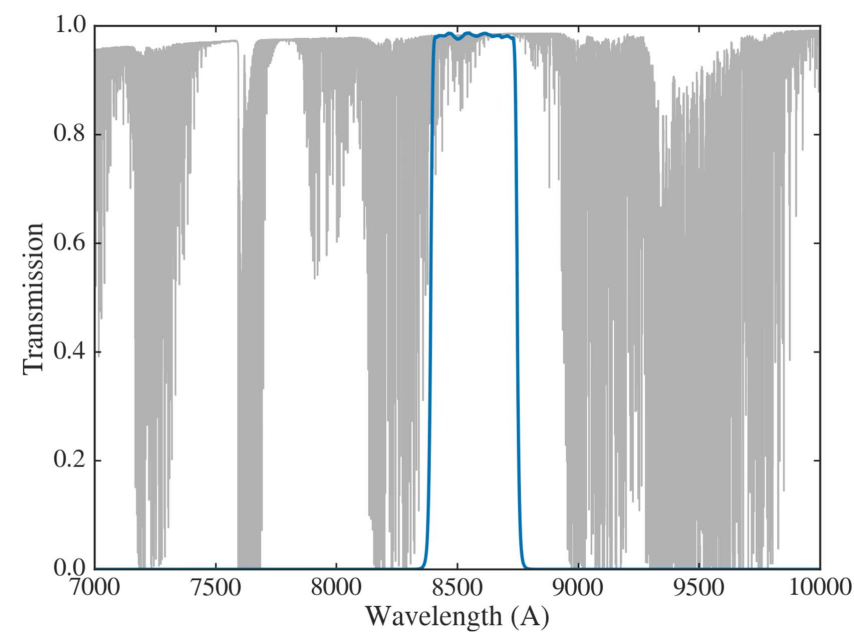

Figure 1. Semrock filter transmission shown in blue. Shown in gray is the atmospheric transmission calculated using TERRASPEC (Bender et al. 2012). Filter transmission curve from the Semrock Web site (see the text for details).

\subsubsection{Scintillation}

For bright nearby stars, the photometric precision is often not limited by photon noise or by background sky counts, but rather by intensity fluctuations-or scintillation-produced by Earth's atmosphere (Osborn et al. 2015). Scintillation is caused by the spatial intensity fluctuations crossing the pupil boundary, and the timescale is determined by the wind speed of the turbulent layer (Young 1967; Dravins et al. 1998; Osborn et al. 2011).

The expected scintillation noise for a given star is described by Young (1967) and Dravins et al. (1998) in units of relative flux, with the following approximation:

$$
\sigma_{s}=0.09 D^{-\frac{2}{3}} \chi^{1.75}\left(2 t_{\mathrm{int}}\right)^{-\frac{1}{2}} e^{\frac{-h}{h_{0}}},
$$

where $D$ is the diameter of the telescope in centimeters, $\chi$ is the airmass of the observation, $t_{\text {int }}$ is the exposure time in seconds, $h$ is the altitude of the telescope in meters, and $h_{0} \simeq 8000 \mathrm{~m}$ is the atmospheric scale height. The constant 0.09 factor in front has units of $\mathrm{cm}^{2 / 3} \mathrm{~s}^{1 / 2}$, giving the scintillation error in units of relative flux. This equation is approximate and highly reliant on the site and the strength and direction of winds in the upper atmosphere, and the exponent above the airmass term can range from 1.5 to 2.0 , depending on the wind direction (Southworth et al. 2009; Osborn et al. 2011). However, for exposures longer than $1 \mathrm{~s}$ (long exposure regime for scintillation), the wind profile tends to average out (Kornilov 2012; Osborn et al. 2015). Additionally, it has been suggested by Osborn et al. (2015) that the median value of scintillation is a factor of 1.5 higher than suggested by Equation 5. In the case of differential photometry, the strength of scintillation depends on the number of uncorrelated reference stars $n_{E}$ (Kornilov 2012). The degree of correlation depends on the angular separations of stars from each other (Kornilov 2012), where $20^{\prime \prime}$ is generally the radius within which they are correlated. Combining these two terms and assuming our target and reference stars are uncorrelated, we have the following equation for the scintillation for 


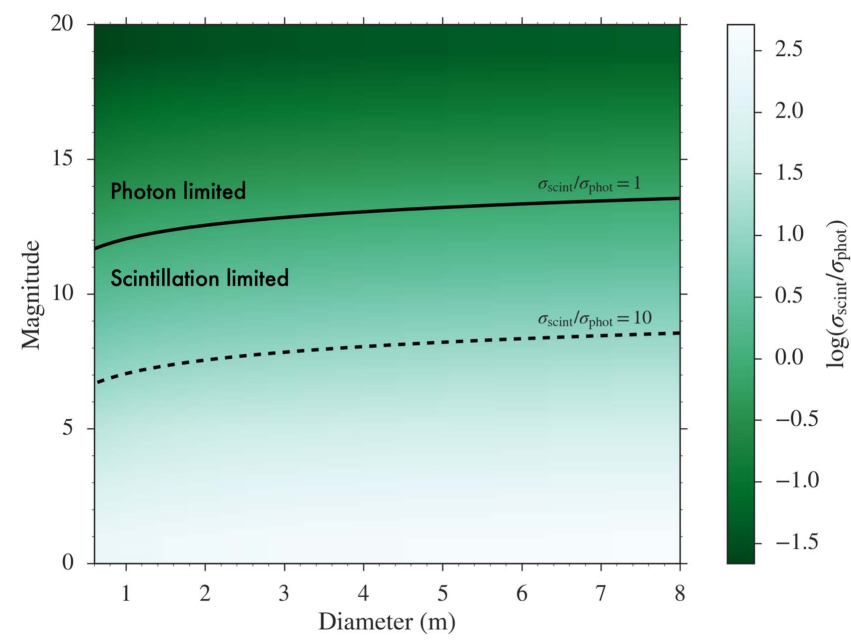

Figure 2. Ratio of scintillation noise to photometric noise in the magnitudetelescope-diameter plane, assuming a perfect telescope ( $100 \%$ throughput) and airmass of 1 . The solid curve shows where the scintillation noise equals the shot noise. Therefore, stars below this line are scintillation limited, and above it photon limited. The dashed curve shows where the scintillation noise is an order of magnitude larger than the shot noise. Figure adapted from Osborn et al. (2015).

differential photometry,

$$
\sigma_{\text {scint }}=1.5 \sigma_{s} \sqrt{1+1 / n_{E}}
$$

assuming $n_{E}$ uncorrelated reference stars. This illustrates the advantages of using multiple reference stars for precision photometry. The total error including scintillation is then

$$
\sigma_{\text {tot }}=\sqrt{\sigma_{\text {rel flux }}^{2}+\sigma_{\text {scint }}^{2}},
$$

assuming that the other errors, e.g., from transparency variations and differential extinction, are minimal.

As the photon and scintillation errors tend to be the largest sources of noise in ground-based photometry, it is instructive to look at the ratio of the two, $\sigma_{\text {scint }} / \sigma_{\text {phot }}$, to see when each dominates. To do this, we adapt the calculation and methodology similar to those described by Osborn et al. (2015), showing the dependence of this ratio on the target star magnitude and telescope diameter plane (Figure 2). The pure photon noise in normalized relative flux units, $\sigma_{\text {phot }}=1 / N$, is calculated using Equation (1) (see also Equation (4)), assuming no sky background and a perfect detector (no read or dark noise) on a telescope with $100 \%$ throughput, and the scintillation error is calculated using Equation (6), assuming an airmass of 1.0, an altitude of $2700 \mathrm{~m}$, and one reference star. For other observational parameters, the results must be scaled accordingly. The solid black line in Figure 2 shows where the scintillation and shot noise errors are equal. Therefore, stars below this curve $\left(\sigma_{\text {scint }} / \sigma_{\text {phot }}<1\right)$ are scintillation limited, and stars above this curve $\left(\sigma_{\text {scint }} / \sigma_{\text {phot }}>1\right)$ are photon limited. The dotted curve shows where the scintillation error is an order of magnitude larger than the photon noise $\left(\sigma_{\text {scint }} / \sigma_{\text {phot }}=10\right)$. The dependence of this ratio with diameter is $\sim D^{1 / 3}$, so the ratio will increase modestly with telescope aperture, but we stress that both error terms, scintillation and photon noise, decrease with telescope aperture as $\sim D^{-2 / 3}$ and $\sim D^{-1}$, respectively. Due to this modest dependence on telescope diameter, as mentioned by Osborn et al. (2015), we can say that stars
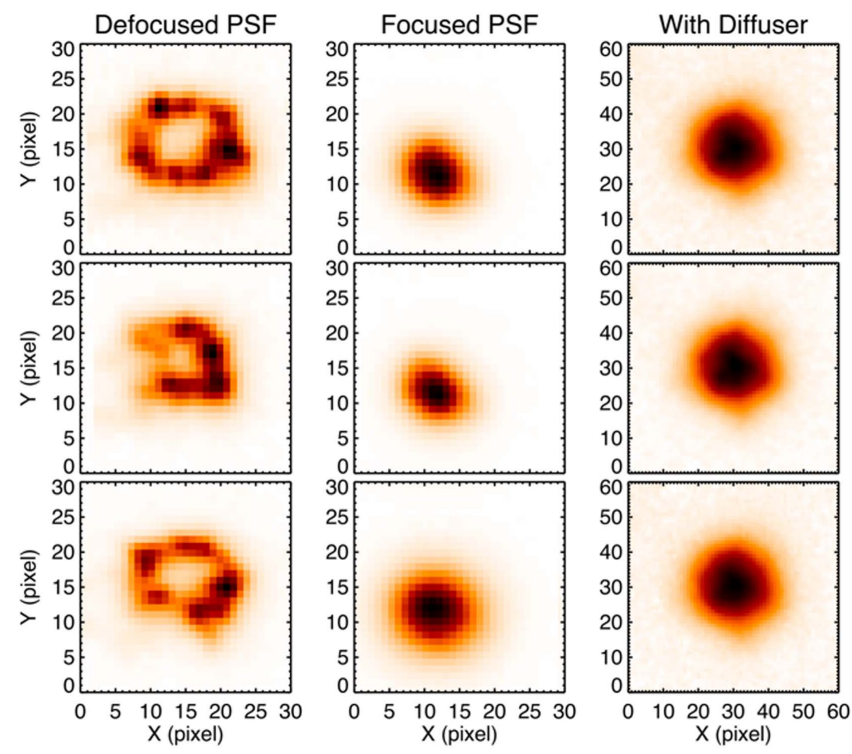

Figure 3. Comparison of Palomar/WIRC PSFs under three observing modes at different epochs: defocused, focused, and diffused. The defocused PSF shows bright spots due to astigmatism of the telescope, inducing a significant amount of "red noise" in the light curve. Both defocused and focused modes show varying PSFs due to seeing variations, while the diffused PSF stays stable in shape (flux level still varies due to telluric fluctuations). For clarity, the images are shown with the diffuser at different scales.

(An animation of this figure is available.)

brighter than a $V$-band mag of $\sim 13$ will be scintillation-limited across different telescopes. 19

\subsection{Reaching High Photometric Precisions}

To reach high photometric precision requires that we consider all of the parameters discussed above. Our overall strategy follows many of the CCD photometry practices common in the field (see, e.g., discussion by Mann et al. 2011). As mentioned by Mann et al. (2011), signals of $\sim 10^{7}$ or higher are needed to achieve submillimag precisions. Spreading the PSF over a large number of pixels is a well-established observing technique to reach such signal levels in a single exposure. Spreading out the light increases exposure times before saturation while simultaneously reducing scintillation errors and flat-field errors due to variations in inter-pixel sensitivities. However, spreading out the light over many pixels increases background noise, which can be the dominant noise source on faint targets and targets observed at redder wavelengths where the background sky is brighter.

This has successfully been done on the ground by telescope defocusing (e.g., Southworth et al. 2009), where the PSF is spread over many pixels through imaging the telescope pupil. However, defocusing often results in a "doughnut"-shaped PSF that is location-dependent across the imaging array, revealing numerous other optical aberrations (Howell et al. 2003). In particular, a defocused image is subject to atmosphere-induced phase errors (seeing) in ways that an in-focus image is not (Figure 3). In both cases, phase errors from seeing will induce fluctuations across the face of the image, and even if the total flux might be conserved, the flux will be redistributed between

\footnotetext{
9 Similar to Osborn et al. (2015), we only plot telescope diameters up to $8 \mathrm{~m}$, as the scintillation relation in Figure 14 is most accurate for this range of telescope diameters.
} 
pixels, producing uncertainties to the extent that the pixel responses are not perfectly calibrated (Mann et al. 2011). For defocused images, these phase-induced errors can create uneven signal distributions across the PSF, often resulting in time-varying high-intensity spikes (column 1 in Figure 3). These spikes cause the PSF to be asymmetric, induces more pixel-dependent errors, and can cause the detector to saturate. The time-varying PSF asymmetry can also cause the centroid of the PSF to shift, reducing photometric precision. For focused observations, the same seeing effect is present, but in this case, the phase errors are more localized around the center of the PSF (instead of being spread out over a doughnut), resulting in a broadened and blurred PSF instead (Figure 3). Despite these drawbacks of defocused images, defocused observations generally yield better photometric precisions than in-focused observations, due to the low dynamic range and high susceptibility to guiding and flat-field errors for in-focused observations.

An "in-focus" diffused image brings out the best from both of these methods, allowing for a high dynamic range and minimal flat-field and guiding errors, while minimizing any phase-induced errors due to seeing. This is illustrated in Figure 3, which compares (a) the defocused and (b) focused PSFs of WIRC to (c) diffused-assisted observations with WIRC (where a "focused" image is deterministically spread out over many pixels). From Figure 3, we see that the defocused PSF changes significantly due to seeing variations (more so than the focused observations, which vary and blur as well), while the diffused PSF is broad and stable throughout the observations. In particular, the defocused PSF shows numerous peaks whose locations and intensities change with time. An animation of this figure is available.

\section{Description of Diffusers}

"Diffusers" is a generic term encompassing optical components or materials that use microscopic surface or bulk structures to control, shape, and homogenize the distribution of light. By precisely controlling the size, shape, location, and distribution of the surface structures used, an input beam can be molded to produce a desired output pattern with broadband compatibility. Diffusers have a plurality of applications, including, but not limited to, use in the telecommunication and automotive industries, and architectural lighting applications. In this paper, we show that diffusers are also applicable for use in precise photometry.

\subsection{Diffuser Types}

Below we give an overview of four basic types of diffusers: ground-glass, holographic, diffractive, and Engineered Diffusers $^{\mathrm{TM}}$.

\subsubsection{Ground-glass Diffusers}

Ground-glass diffusers are the simplest of the four diffuser types discussed here. These diffusers are generally produced by sand-blasting glass using various grit sizes to create small randomized surface features. As the surface features are randomized, ground-glass diffusers offer little control over their diffusing characteristics, resulting in limited control on angular divergence. As such, these diffusers are only capable of producing Gaussian intensity profiles. Although commercially available at low cost, these diffusers have low optical transmission efficiencies and diffuse light at large angles. Ground-glass diffusers with opal coatings can be made to achieve close to Lambertian diffusion.

\subsubsection{Holographic Diffusers}

Holographic diffusers rely on the holographic recording of a speckle pattern on the diffuser substrate. This speckle pattern creates pseudo-random semi-periodic surface structures that can be controlled in a statistical sense, offering precise control over the angular distribution of the output light. These types of diffusers are available from many vendors, including Edmund Optics and, notably, Luminit LLC, which offers holographic Light Shaping Diffusers@, which can be made to have very high transmission efficiencies of over $92 \% .{ }^{20}$ However, as the surface structures are only controlled in a statistical sense, this limits the angular diffusion patterns to be either circular or elliptical, and only offers Gaussian-like intensity profiles (Sales et al. 2004). This is suboptimal for high-precision aperture photometry, as the Gaussian profile has broad and extensive wings, spreading out the signal outside the photometric aperture. Furthermore, Gaussian-shaped PSFs have significant slopes across the full PSF except in the very center, making them more subject to guiding errors and changes in seeing.

\subsubsection{Diffractive Diffusers}

Diffractive diffusers are based on fabricating a phase mask for a single central wavelength and can be made to have efficiencies between $80 \%$ up to $90 \%-95 \% .^{21}$ However, the output pattern is highly sensitive to the wavelength of light used, and as such, these diffusers are largely limited to monochromatic applications in laser systems, but could, however, have possible applications in narrow-band astronomical studies. Due to their high sensitivity with wavelength, we did not study these types of diffusers further for our broadband photometry applications.

\subsubsection{Engineered Diffusers ${ }^{T M}$}

Unlike the other types of diffusers that only offer statistical control of the surface features, diffusers that precisely control the shape, size, and location of its surface features in a deterministic manner have the highest degree of control over their output. Such diffusers, capable of molding the output to a desired intensity profile and light distribution pattern, are now commercially available. We worked closely with RPC Photonics in Rochester, NY, to test and design the diffusers used in this paper. These Engineered Diffusers ${ }^{\text {TM }}$ offer precise beam-control capabilities and utility for many applications.

Engineered Diffusers $^{\mathrm{TM}}$ (Morris \& Sales 2006) are composed of individually manipulated unit cells or microlenslets (Figure 4). By precisely controlling the design and manufacturing processes, a surface can be engineered to produce a desired intensity profile and light distribution pattern for a given input beam. To ensure that the diffuser output is stable toward varying beam input, the size, shape, and location of the microlenslets are varied according to a pre-defined probability distribution chosen to implement the desired beam-shaping

\footnotetext{
${ }^{20}$ See holographic Light Shaping Diffusers at the Luminit LLC Web site: http://www.luminitco.com/.

${ }^{21}$ See, e.g., the discussion on diffractive diffusers at the RPC Photonics Web site: http://www.rpcphotonics.com/product/diffractive-optics/.
} 


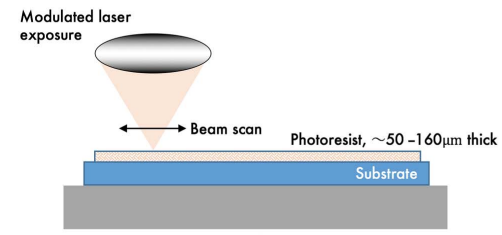

a)

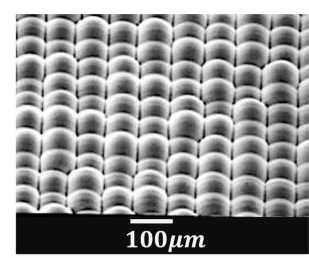

b)
Figure 4. (a) Schematic diagram of the laser writing process: a modulated laser beam is scanned across a surface to deterministically write in surface features. (b) Surface electron microscope image of the surface of an Engineered Diffuser ${ }^{\mathrm{TM}}$, demonstrating a deterministic placement of surface features and microlenslets. Image used with permission from RPC Photonics.

functions (Sales et al. 2004). Additionally, this microlens distribution can be carefully designed to avoid discontinuities and minimize scattered light and diffraction artifacts from the output. In this manner, Engineered Diffusers ${ }^{\mathrm{TM}}$ retain the best properties of both random and deterministic diffusers.

The diffusers we used are manufactured by RPC Photonics using a proprietary laser writing process (Figure 4). The process starts by making a master, consisting of a substrate coated with a thick layer of photoresist. A focused UV laser beam is scanned across the surface, and by modulating the intensity of the laser beam, different exposures can be achieved, which breaks down the photoresist. By developing out the exposed areas, a deterministically structured surface with controlled size, shape, and depth is produced (Figure 4(b)). The master can then be used to produce submasters and replicas in different materials, including fused silica, silicon, or on polymer layers on top of glass, allowing these diffusers to span a wide application wavelength range from $193 \mathrm{~nm}$ to $10.6 \mu \mathrm{m}$ (Sales et al. 2004).

Although the details of the exact design and manufacture of these structures are proprietary to RPC Photonics, a few general design rules are noteworthy.

1. Designing and fabricating diffusers with larger angles of diffusion is easier than those smaller angles, if a top-hat like shape with a steep fall-off is desired.

2. Polymer diffuser patterns bonded to glass substrates (like those we tested at the APO $3.5 \mathrm{~m}$ ) are less expensive since the laser writing process can be used to make and replicate them. Pure fused silica diffusers (like those we tested at Palomar) are made with a process similar to a reactive ion etch and are more time consuming and expensive.

3. Discontinuities in the surface structures can lead to additional scattered light. This can be mitigated in the design process if the requirements are well stated.

4. Systematic periodic errors or shifts in the laser writing process can result in the diffuser pattern being gratinglike, diffracting light at very low efficiencies into non-zero orders. This was discovered with tests on the ARC $3.5 \mathrm{~m}$ (see Section 7.2). This can also be mitigated in future designs (RPC Photonics 2017, private communication).

\subsection{Using Diffusers in Telescopes for Precise Photometry}

Due to their light-shaping and beam-homogenizing capabilities, beam-shaping diffusers are attractive optical devices for use in high-precision photometry applications. In the ideal case, such a diffuser would create a top-hat PSF shape with steep sides and a flat top subtending many tens of pixels in diameter, minimizing the signal lost outside the photometric aperture. Furthermore, like mentioned above, a top-hat PSF is more favorable than, e.g., a Gaussian-shaped PSF, which has significant slopes everywhere except in the very center, making every pixel subject to guiding errors and changes in seeing. Meanwhile, a top-hat PSF restricts guiding errors due to PSF slopes to only the edge pixels because the inner pixels see the same flux regardless. To enable the adoption of a beam-shaping diffuser over a broad range of astronomical instrumentation and allow for maximum flexibility, the diffuser should work in both converging and collimated beams.

Figure 5 shows a schematic of a diffuser in a converging telescope beam. Light from a star (a) arrives at the telescope (b) as a collimated beam. In this schematic, the telescope acts as a lens with an effective focal length $f$. The diffuser, with an opening angle of $\theta$, is placed in the converging beam at a distance $D$ from the detector image plane. The diffuser pattern faces the incoming starlight. The approximate FWHM of the resulting diffused spot on the image plane is given by

$$
S=D \tan \theta .
$$

By using different distances from the diffuser to the focal plane, the size of the resulting PSF can be tuned. In most telescope systems, this translates into a relatively small opening angle $\theta$, on the order of $0.05-0^{\circ} .5$. However, we also tested angles as large as $2^{\circ}$ with excellent photometric performance.

\section{Lab Setup and Diffuser-assisted Observations}

\subsection{Diffuser Characterization Lab Setup}

To study diffuser PSFs in collimated and converging beams, and how their PSF changes with distance, we set up a dedicated test bench (Figure 6). A single-mode fiber was coupled to a collimating lens system, composed of two identical 2 inch diameter $f / 6.3$ camera lenses. Although the coupling of a single-mode fiber to a broadband light source is inefficient, this posed no issues for our experiment. To better match the properties of incoherent starlight on sky, we explicitly avoided using a coherent supercontinuum source in our lab setup in favor of a low-coherence broadband source (Alphabright Quartz-Tungsten-Halogen light source). The diffusers were mounted on a custom-made rotation mount at a varying distance $D$ from an FLI Proline PL4710-1 MB monochrome $1056 \times 1027$ CCD camera with $13 \times 13$ micron pixels. Before mounting the diffuser, the test bench was aligned to ensure the optical system was properly focused. The rotation mount used a stepper motor driven by an Arduino, capable of rotating the diffuser at $1-2 \mathrm{~Hz}$. This enabled us to smooth out the PSF for exposure times of a few seconds, as is discussed further in Section 5.2.2. The exposures were dark-subtracted, using a master median dark built from a sequence of 25 dark frames.

\subsection{Diffusers Used}

Table 2 summarizes the different diffusers tested in this paper. Most of the diffusers were Engineered Diffusers ${ }^{\mathrm{TM}}$ fabricated by RPC Photonics. Additionally, we tested a holographic diffuser from Edmund Optics with a $1^{\circ}$ opening angle. However, being a holographic diffuser, it had a Gaussian intensity profile with broad extensive wings, which is suboptimal for precise aperture photometry. Instead, we focused further tests on the three top-hat off-the-shelf diffusers from RPC Photonics with opening angles 
(a) Star

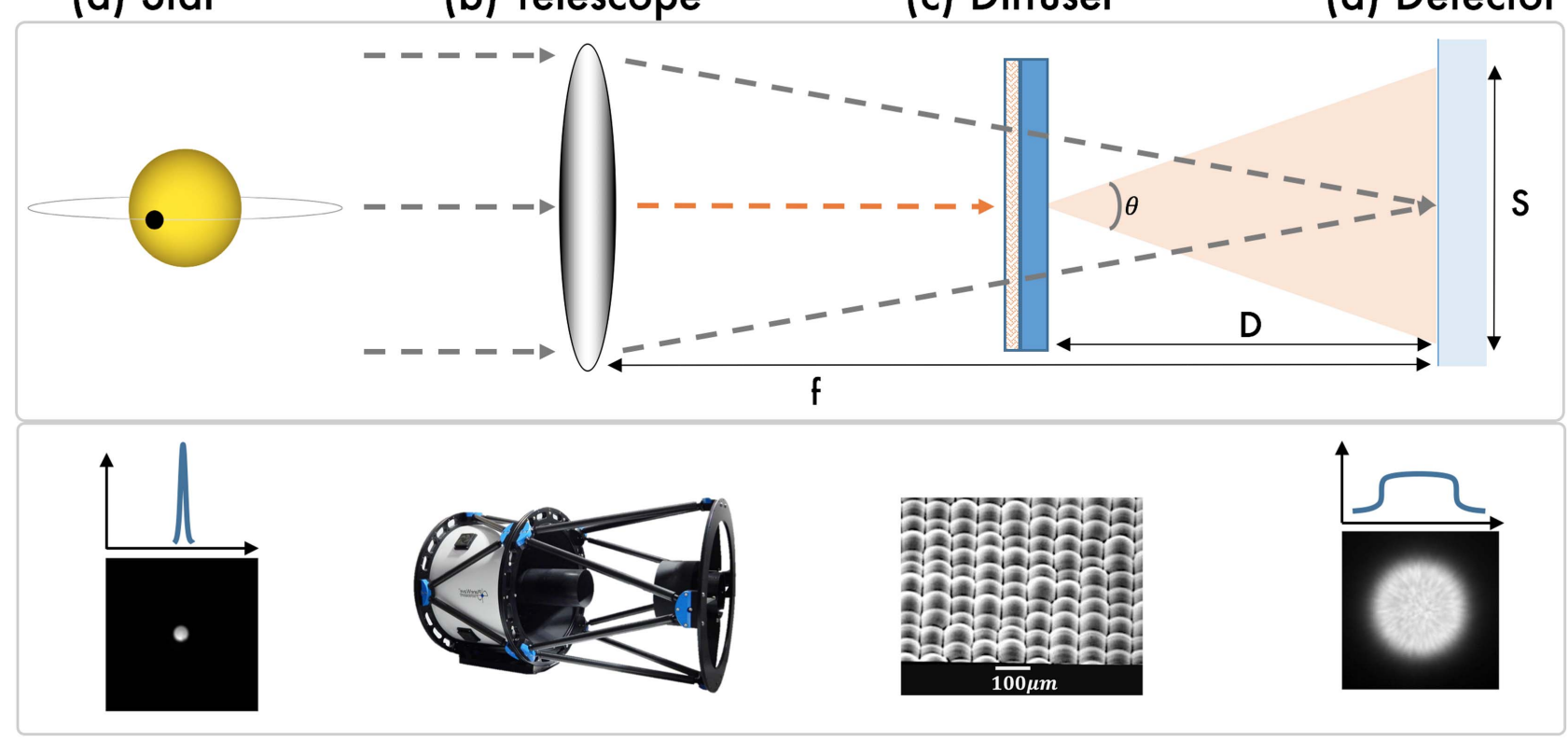

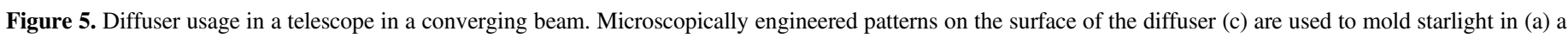
converging beam (b) to a broad and stable top-hat shape on the detector (d). Diffuser surface structures: image credit RPC Photonics.

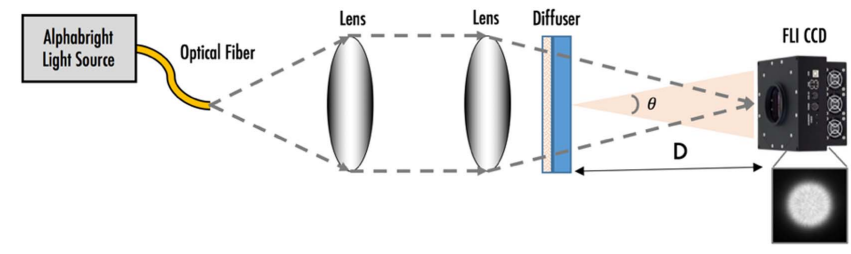

Figure 6. Lab test setup to characterize diffusers in converging and collimated beams.

of $0.25,0.5$, and 2.0 , respectively. The off-the-shelf diffusers from RPC were not optimized for our application. Therefore, we worked with RPC to fabricate a custom diffuser optimized for installation in ARCTIC, with the specifications informed by lab tests of off-the-shelf diffusers. Specifically, we optimized the customized diffuser to give a top-hat PSF with a $10^{\prime \prime}$ FWHM on the ARCTIC detector array, resulting in a diffuser angle of $0^{\circ} .4$. The final diffuser opening angle as fabricated was $\sim 0{ }^{\circ} 34$.

Our NIR diffuser was designed for the WIRC at the Palomar 200 inch Hale telescope. WIRC sits at the prime focus of the telescope and consists of a collimation assembly that collimates the beam coming from the primary, followed by a Lyot stop and two filter wheels tilted by $7^{\circ}$ to minimize ghost reflections. Because the filter wheel assembly in WIRC is located in the collimated beam, we chose to install the diffuser directly into one of the filter slots inside the WIRC cryogenic dewar for minimal modification to the instrument.

Our NIR diffuser was designed to satisfy four conditions: (1) the FWHM of the diffused PSF should be large enough to spread starlight onto a large number of pixels for the purpose of mitigating inter- and intra-pixel variations, (2) the FWHM of the diffused PSF needs to be larger than that of the seeing PSF to ensure that the diffused PSF does not change significantly under variable seeing conditions, (3) the FWHM also needs to be small enough such that stars of $\sim 11-12 \mathrm{mag}^{22}$ in $K_{S}$ are not

\footnotetext{
${ }^{22}$ Based on the typical brightness of faint reference stars in our target field.
}

limited by background noise, and (4) the FWHM needs to be small enough to avoid cross-talk in PSFs for neighboring stars. Given that the typical seeing at the Hale telescope is between 0 ". 5 and 1 ".5, we thus designed the diffuser to have a top-hat PSF with FHWM of $3^{\prime \prime}$ to meet the above conditions. Because the filter wheels are inside the cryogenic dewar, the diffuser is made of fused silica for its low thermal expansion coefficient. The size is $60 \mathrm{~mm} \times 5 \mathrm{~mm}$, compatible with the filter wheel in the camera. The working wavelengths of the diffuser were optimized to cover the NIR $J, H$, and $K$ bands.

\subsection{On-sky Diffuser-assisted Observations}

Table 3 describes the three observatories used to perform diffuser-assisted photometry in this work. The observations for each are further discussed below.

\subsubsection{Penn State PlaneWave CDK 24 inch Telescope}

To verify the operation of diffusers, we tested various diffusers on-sky using the Penn State PlaneWave 24 inch telescope. The telescope was installed in 2014 at Davey Lab Observatory in University Park, PA, at an altitude of $360 \mathrm{~m}$ above sea level. The telescope has an Apogee/Andor Aspen CG 42 camera, using a CCD42-10 $2048 \times 2048$ pixel chip from E2V with 13.5 micron pixels. This results in a plate scale of $\sim 0$ ". 77 pixel $^{-1}$ and an FOV of $24^{\prime} \times 24^{\prime}$. The telescope is equipped with a dual filter wheel (AFW50-10S dual filter wheel) capable of housing $202^{\prime \prime} \times 2^{\prime \prime}$ filters. The diffuser was placed in the filter wheel closer to the camera, so that the diffuser was $50 \mathrm{~mm}$ away from the detector. Using a dual filter wheel allowed us to easily perform diffuser-assisted observations in different filters.

$55 \mathrm{Cnc}-\mathrm{As}$ an illustration of our observations with Penn State's CDK 24 inch telescope, we discuss our out-of-transit observations of $55 \mathrm{Cnc}$ using a 2.0 off-the-shelf diffuser on this telescope. $55 \mathrm{Cnc}$ is a nearby bright ( $V=5.95 \mathrm{mag}) \mathrm{G} 8 \mathrm{~V}$ binary star (its companion $55 \mathrm{Cnc} \mathrm{B}$ is an M4.5V dwarf). We 
Table 2

Summary of the Different Diffusers Tested and Used in This Work

\begin{tabular}{|c|c|c|c|c|}
\hline Opening Angle & Company & Diffuser Part Number & Type & Size \\
\hline 1.0 & Edmund Optics & $\# 47-990$ & Holographic Diffuser & $2^{\prime \prime} \times 2^{\prime \prime}$ \\
\hline 0.25 & RPC Photonics & EDC-0.25-07118-A-2S & Off-the-shelf Engineered Top-hat Diffuser ${ }^{\mathrm{TM}}$ & $2^{\prime \prime} \times 2^{\prime \prime}$ \\
\hline 0.5 & RPC Photonics & EDC-0.5-07101-A-2S & Off-the-shelf Engineered Top-hat Diffuser ${ }^{\mathrm{TM}}$ & $2^{\prime \prime} \times 2^{\prime \prime}$ \\
\hline 2.0 & RPC Photonics & EDC-2-07331-A-2S & Off-the-shelf Engineered Top-hat Diffuser ${ }^{\mathrm{TM}}$ & $2^{\prime \prime} \times 2^{\prime \prime}$ \\
\hline 0.34 & RPC Photonics & Custom & Custom Engineered Top-hat Diffuser ${ }^{\mathrm{TM}}$ & $150 \mathrm{~mm}$ circle \\
\hline 0.08 & RPC Photonics & Custom & Custom Engineered Top-hat Diffuser ${ }^{\mathrm{TM}}$ & $60 \mathrm{~mm}$ circle \\
\hline
\end{tabular}

used 53 Cnc, a nearby bright M3III star $(V=6.23)$, $\sim 4$.5 away from our target, as our main reference star. We observed the system in Johnson $I$, as both stars are well matched in brightness in that filter, and to minimize the impact from the Moon brightness, which was at $\sim 88 \%$. The observations were done on 2016 March 27, from 04:00 UT to 06:30 UT. The target was setting, and starting at airmass 1.16, ending at airmass 1.9. The conditions were good, with few to no clouds. The 2.0 diffuser allowed us to spread the PSF over $\sim 120^{\prime \prime}$. Without the diffuser, the detector saturated almost instantly.

The exposure time was $120 \mathrm{~s}$, with $11 \mathrm{~s}$ of dead time between exposures. This allowed us to collect $>10^{8}$ counts in the target and reference stars. We took 20 dome flats and 20 dark frames, and median-combined them using AstroImageJ (Collins et al. 2017) to create master dark and flat frame images. We used AstroImageJ for the photometric reduction. The aperture setting that gave the smallest residuals was 100, 150, and 200 pixels for the aperture radius, and the inner and outer annuli, respectively. To arrive at the final light curve, we detrended the raw data with airmass, a straight line, and $x$ and $y$ pixel centroid coordinates using the detrend function in AstroImageJ.

\subsubsection{Apache Point $3.5 \mathrm{~m}$ Telescope}

In 2016 September, we installed a custom Engineered Diffuser $^{\mathrm{TM}}$ as part of the Astrophysical Research Consortium Telescope Imaging Camera (ARCTIC) on the ARC $3.5 \mathrm{~m}$ Telescope at Apache Point Observatory. ARCTIC uses a back-illuminated STA4150LN BI $4096 \times 4096$ pixel CCD with 15 micron pixels. This gives an unbinned plate scale of 0 . 114 pixel $^{-1}$ and a field of view of $7 ! 5 \times 7 ! 5$. The detector has four amplifiers and can be read out using one amplifier (lower left) or using all amplifiers simultaneously dividing the frame into four quadrants.

Figure 7(a) shows an image of the final diffuser at ARCTIC, along with the dedicated holder and rotator we designed. The holder is capable of sliding the diffuser in and out of the telescope beam and rotating the diffuser during observations with a pneumatic motor that moves with the diffuser holder assembly. The holder places the diffuser in front of the ARCTIC six-position filter wheel and $200 \mathrm{~mm}$ away from the detector plane, creating a top-hat PSF with $\sim 9$ " FWHM. Figure 7(b) shows a schematic diagram of the diffuser location in ARCTIC, where the rays are traced with no diffuser in the beam path. The inset shows a footprint diagram of the beam at the diffuser location: an annulus due to the central obstruction of the telescope. These parameters - the size of the beam and the distance from the detector plane-were the key parameters in the optimization process of the diffuser.
For some of our on-sky observations with ARCTIC and the diffuser, we used an off-the-shelf narrow-band filter from Semrock operating in a band with minimal water absorption centered at $857 \mathrm{~nm}$ (Figure 1). However, the filter was only $2^{\prime \prime} \times 2^{\prime \prime}$ in size, truncating the FOV from $8^{\prime} \times 8^{\prime}$ to about $180^{\prime \prime} \times 180^{\prime \prime}$. Therefore, our on-sky tests of the diffuser in this filter were limited to bright, closely separated targets. We did not observe any noticeable fringing effects in this filter with ARCTIC, which is commonly seen with redder filters such as SDSS $z^{\prime}$.

16 Cygni-Using ARCTIC with a diffuser, we observed 16 Cygni, a nearby bright G-dwarf binary star, where 16 Cyg A and $\mathrm{B}$ have $V$ magnitudes of 5.95 and 6.2, respectively, and a separation of 39". 5 . The observations were performed on 2016 September 20 from 2 am to 6 am UT. The target rose during the observations, starting at airmass 1.07 , peaking at 1.05 at the meridian, and ended at 1.30 at the end of the observations. Moon illumination was $\sim 85 \%$. The night was not photometric, with variable seeing $>2^{\prime \prime}$ FWHM and intermittent clouds. Nevertheless, due to the flux-homogenizing properties of the diffuser, the PSF remained stable throughout the night. To maximize observing efficiency and minimize scintillation noise, the detector was configured to use one amplifier in fast readout mode, reading out only a subframe of $783 \times 813$ pixels encompassing $16 \mathrm{Cyg} \mathrm{A}$ and the reference star $16 \mathrm{Cyg}$ B. This resulted in a short readout time of $5 \mathrm{~s}$. Binning was set to $1 \times 1$ due to the brightness of the target, resulting in an exposure time of $16 \mathrm{~s}$ to reach $\sim 40,000$ peak counts per pixel for $16 \mathrm{Cyg} \mathrm{A}$, and thus a total observing cadence of $21 \mathrm{~s}$ and duty cycle of $76 \%$.

Our data reduction consisted of standard aperture photometry performed with the AstroImageJ software suite. After experimenting with different aperture radii and annuli, the best rms precision was achieved with an aperture radius of 100 pixels, and inner and outer annulus radii of 100 and 200 pixels, respectively. For calibration, a set of 25 bias frames were used, along with 25 dome flats. Each set was median-combined using AstroImageJ.

16 Cygni A and B were observed by Kepler in both shortand long-cadence mode, and in this paper, we compare our precision achieved with ARCTIC to that achieved with Kepler. There have been previous studies in the literature using Kepler data of 16 Cygni $\mathrm{A}$ and $\mathrm{B}$, using short-cadence data for astroseismology (e.g., Metcalfe et al. 2012; Lund et al. 2014) and gyrochronology (Davies et al. 2015), and long-cadence data to study the link between radial velocity variations and photometric flicker (Bastien et al. 2014). 16 Cygni A and B have Kp magnitudes of 5.864 and 6.095, respectively, and Kepler Input Catalog (KIC) IDs of KIC12069424 and KIC12069449, respectively. Due to their brightness, both stars are well above the saturation limit of Kepler, which is $K_{p} \sim 11.5$ (Gilliland et al. 2011). Still, excellent precision 
Table 3

Summary of the Different Telescopes where We Have Tested Diffusers on Sky

\begin{tabular}{|c|c|c|c|c|}
\hline & Parameter & CDK 24 & APO & Palomar \\
\hline General & $\begin{array}{l}\text { Telescope } \\
\text { Instrument } \\
\text { Beam } f / \# \text { at diffuser } \\
\text { Detector } \\
\text { Wavelengths }\end{array}$ & $\begin{array}{l}\text { PlaneWave CDK } 24 \\
\text { Apogee Aspen CG } 42 \\
f / 6.3 \\
\text { CCD } \\
\text { Optical }\end{array}$ & $\begin{array}{l}3.5 \mathrm{~m} \text { ARC } \\
\text { ARCTIC } \\
f / 8.0 \\
\text { CCD } \\
\text { Optical }\end{array}$ & $\begin{array}{l}\text { Hale } 200 \text { inch } \\
\text { WIRC } \\
\text { Collimated } \\
\text { Hawaii-2 } \\
\text { NIR } J, H, K\end{array}$ \\
\hline
\end{tabular}

levels can be achieved for saturated stars in the Kepler data, due to the conservation of charge in the Kepler CCDs, by summing up the counts in the surrounding pixels, commonly yielding precisions down to $40 \mathrm{ppm}$ for stars $\sim 7 \mathrm{mag}$ (Gilliland et al. 2010). The data are easily retrievable from MAST. ${ }^{23}$ Short-cadence data are available for both stars for Quarters 6-15, with the exception of Quarter 13. Quarter 6 has a known problem with its photometric precision as it did not use an optimized aperture in the photometric retrieval, resulting in data of rather poor quality (Lund et al. 2014). Therefore, we focused our comparison to Kepler, by looking at both the shortcadence and long-cadence data from Kepler from Quarters 7-15, excluding Quarter 13.

Transit of WASP-85 A $b$-WASP-85 A b was discovered by Brown et al. 2014 and observed by $K 2$ in Campaign 1 (EPIC 201862715). The star is a G5 dwarf, with a $V$ magnitude of 11.2 and Kp magnitude of 10.247 . WASP-85 A forms a close visual binary (angular separation of 1."5) with a cooler and dimmer $(V=11.9)$ K0 dwarf companion, WASP-85 B. With the diffuser PSF FWHM being $\sim 9^{\prime \prime}$, this close proximity of the two stars causes the stellar PSFs to completely overlap in the diffuser images. Despite the overlapping PSFs, we are able to recover very high photometric precisions, as we discuss in Section 5.2.2.

Our transit observations of WASP-85 A b were performed on January 31 from 08:30 to 13:00 UT. The exposure time was initially set at $7 \mathrm{~s}$. However, the target was rising, starting from airmass 1.20 during the beginning of the observations, peaking at 1.11 , and ending at airmass 1.54 , and the exposure times were reduced to $6 \mathrm{~s}$ after 15 minutes of observations to keep the exposures at $\sim 30,000$ peak counts, well within the linear regime of the detector. This change in exposure time did not result in a visible change in photometric precision. Moon brightness was $\sim 12 \%$. The filter used was SDSS $r^{\prime}$. The binning mode was $4 \times 4$, with the detector read out in quad amplifier and fast readout mode, resulting in a readout time of $2.5 \mathrm{~s}$. We assume a total cadence of $8.5 \mathrm{~s}$, as $\sim 95 \%$ of the images were taken at this cadence, resulting in an observing efficiency of $80 \%$ for these observations.

The data reduction was performed using two independent photometry pipelines. First, we used a photometry pipeline being developed by B. Morris et al. (2017, in preparation), which implements principal component analysis (PCA) to find an optimal set of aperture radii, comparison stars, and environmental measurements used for detrending. The aperture

\footnotetext{
${ }^{23}$ MAST: http://archive.stsci.edu/kepler/data_search/search.php.
}

radius used was 19 pixels, and the radius of the inner and outer radii were 32 and 55 pixels, respectively. The data were independently reduced using AstroImageJ using the same radii and detrending parameters, giving consistent results. A set of three reference stars within the FOV with median fluxes between 0.2 and 1.0 times that of the target were used in the differential photometry. A set of 22 darks and a set of 20 flats were median-combined to create master flat and dark frames. In $4 \times 4$ binning at ARCTIC, a relatively high fraction of cosmic rays and charge particle events is observed in the science frames. To reduce the effect of these events on our photometry, we ran the data through the astroscrappy package, ${ }^{24}$ a cosmic-ray rejection package written in Python, based on the Laplacian-edge cosmic-ray rejection algorithm described by van Dokkum (2001). Using the default parameters in astroscrappy resulted in fewer saturated outliers observed in the light curve.

To compare our best-fit planet parameters with the values reported in the literature, we fit the transit using the Markov Chain Monte Carlo (MCMC) approach described in Section 6.

Transit of TRES-3b-TRES-3b is a hot Jupiter $\left(R \sim 1.3 R_{\text {Jup }}\right)$ discovered by O'Donovan et al. (2007), and is in a $P=$ 1.306 days orbit around a G4 V dwarf star with a $V$ magnitude of 12.4. This target has been well-studied in the literature (e.g., Sozzetti et al. 2009; Knutson et al. 2014). The TRES-3 field has a number of similarly bright reference stars close by (within the ARCTIC FOV for our purposes), which is beneficial for highprecision ground-based differential photometry. Choosing to observe this target thus allowed us to further test the limits of the diffuser-assisted photometry technique with ARCTIC, by observing a clear transit signal on a clear night at good airmasses.

Our observations of this target were performed on 2017 March 12 from 08:45 UT and 12:20 UT, where the target rose during the night, from an airmass of 1.90 to 1.04 . The Moon was full during these observations (brightness $\sim 100 \%$ ), and thus to minimize sky-background noise, we observed the target in SDSS $i^{\prime}$. We used an exposure time of $30 \mathrm{~s}$ with ARCTIC in quad amplifier fast-readout $4 \times 4$ binning mode, resulting in a readout time of $2.5 \mathrm{~s}$. The total cadence was thus $32.5 \mathrm{~s}$, yielding an observing efficiency of $92 \%$.

Similar to the WASP-85 observations above, the data reduction was performed using AstroImageJ, after cleaning the images of cosmic rays and charged particle events using astroscrappy. We used 13 reference stars with a flux

\footnotetext{
$\overline{24}$ astroscrappy is available on Github here: https://github.com/astropy/ astroscrappy.
} 


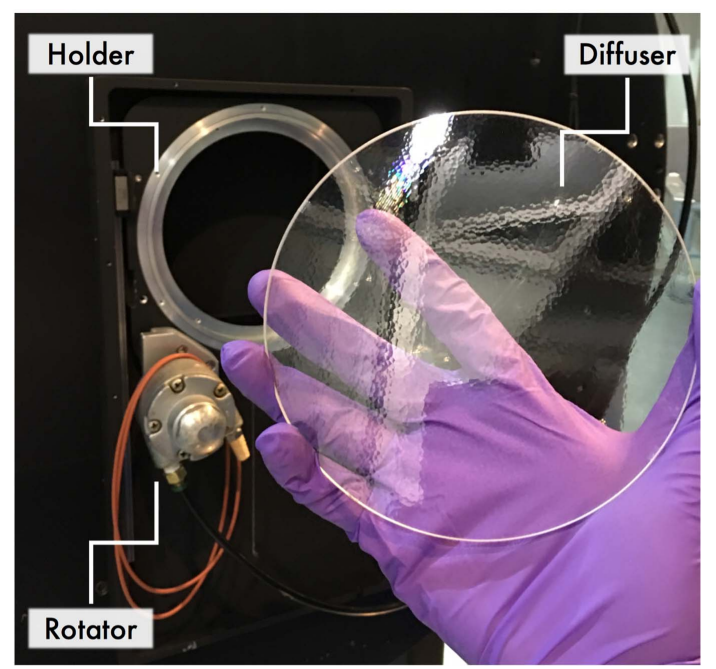

a)

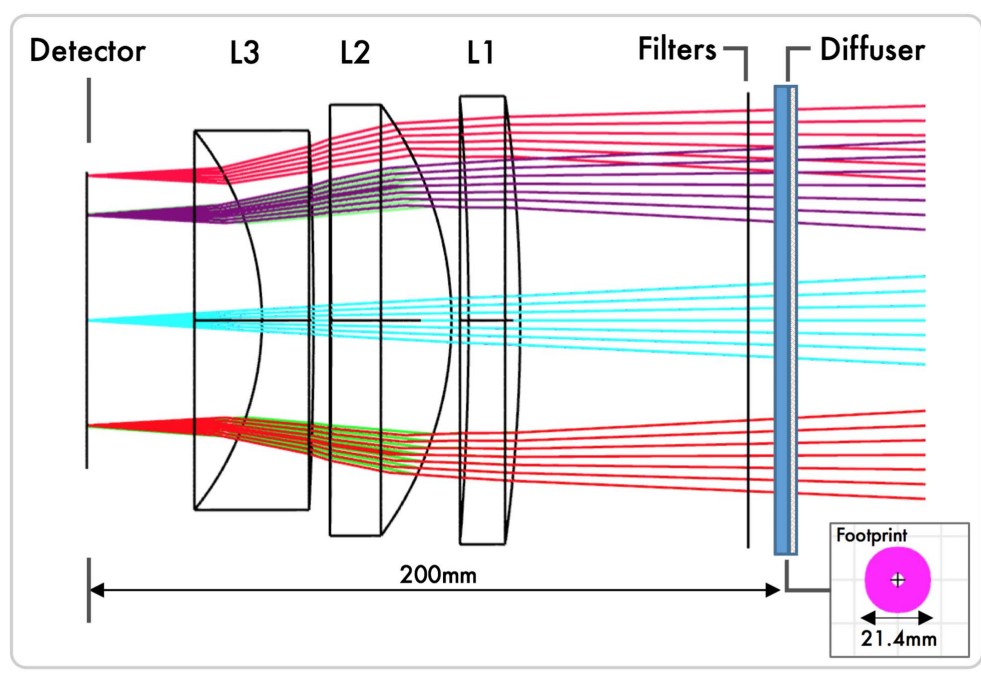

b)

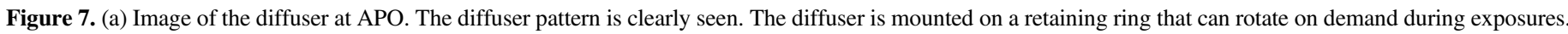

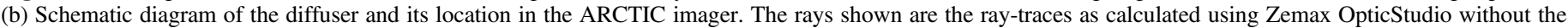
diffuser. The footprint diagram shows the beam footprint at the diffuser location.

between $4 \%$ and $180 \%$ of the flux of the target star. After systematically testing a number of different aperture settings in AstroImageJ, the aperture radius that gave the smallest unbinned rms scatter was 19 pixels, and with inner and outer radii of 32 and 50 pixels, respectively. To create the final light curve, we fit the transit guided by the parameters presented in O'Donovan et al. (2007), performing a simultaneous transit fit and detrending using AstroImageJ, using airmass, a straight line, and $x$-, $y$-centroid pixel coordinates in AstroImageJ.

Similar to the WASP-85 A b transit, to compare our best-fit TRES-3b planet parameters with the values reported in the literature, we fit our TRES-3b observations with the MCMC approach described in Section 6.

\subsection{Palomar Hale/WIRC}

The Palomar experiment started in 2013. RPC delivered the first engineered diffuser with a Gaussian, instead of a top-hat, profile. ${ }^{25}$ We therefore went ahead and installed the diffuser on the WIRC camera in 2013 November for testing observations.

Our on-sky test took place on UT 2013 December 21, using the old science-grade HAWAII-2 array in WIRC with a wide FOV of $8.7 \times 8$.7. Unfortunately, due to a fatal failure (explosive debonding and separation of the semiconductor from its substrate) of the HAWAII-2 array a few months after our first on-sky test, we were not able to conduct additional tests as the replacement array was not science grade, which significantly limited the precision of our photometry due to excessive hot and bad pixels and uneven linearity in different quadrants. Therefore, in this paper, we only demonstrate the performance of our IR diffuser using one night of observation. Since then, in late 2016 and early 2017, WIRC has been retrofitted with a science-grade HAWAII-2 detector, enabling us to continue our efforts in using a new diffuser on sky using the updated WIRC system.

\footnotetext{
25 Another diffuser closer to top-hat shape was remade and delivered within a few months.
}

The observations were carried out in the $K_{S}$ band near the field of XO-3. Because XO-3 $(K=8.8)$ is too bright to have enough reference stars of similar magnitude, we chose a nearby field and a fainter star, 2MASS J04230271 + 5740319 $(K=10.79, \quad V=13.26)$, as our photometry target. The observation lasted for only $\sim 3.5 \mathrm{hr}$ before telescope closure due to critical weather conditions. Nonetheless, these observations still provided a useful test of the diffuser's performance. The telescope was kept focused during the observation, while the diffuser was used to control the PSF. The final diffused PSF had a Gaussian shape with an FWHM of 17.4 pixels (4!" 35) on average. A total of 225 images were recorded continuously with $40 \mathrm{~s}$ exposures, although four images were later rejected due to passing clouds and using one double-correlated sampling (1 Fowler).

Reduction of the images was carried out using our standard WIRC photometry pipeline described in Zhao et al. (2012, 2014). We corrected for time-varying telluric and instrumental effects by selecting 10 reference stars that had median fluxes between 0.3 and 1.0 times that of the target and showed no peculiarities in their light curves. Fainter stars were excluded due to low signal-to-noise ratio, while stars brighter than the target saturated the detector. The $x$ and $y$ positions of the stars' centroids varied by less than 3 pixels, with a standard deviation of 0.63 pixel in $x$ and 0.51 pixel in $y$. The airmass changed from 1.22 at the start to 1.11 at the end. We applied 48 different aperture sizes with a step of 0.5 pixels for the target and reference stars, with the same size aperture used for all stars in each step. The aperture with a radius of 16 pixels $\left(4^{\prime \prime}\right)$ produced the smallest scatter in the reduced light curve and was thus used for subsequent analyses.

\section{Results}

\subsection{Characterizing Diffusers}

\subsubsection{Diffusers Work Similarly in Converging and Collimated Beams}

Because an imaging system inherently has a converging beam before the detector, the most straightforward way to 

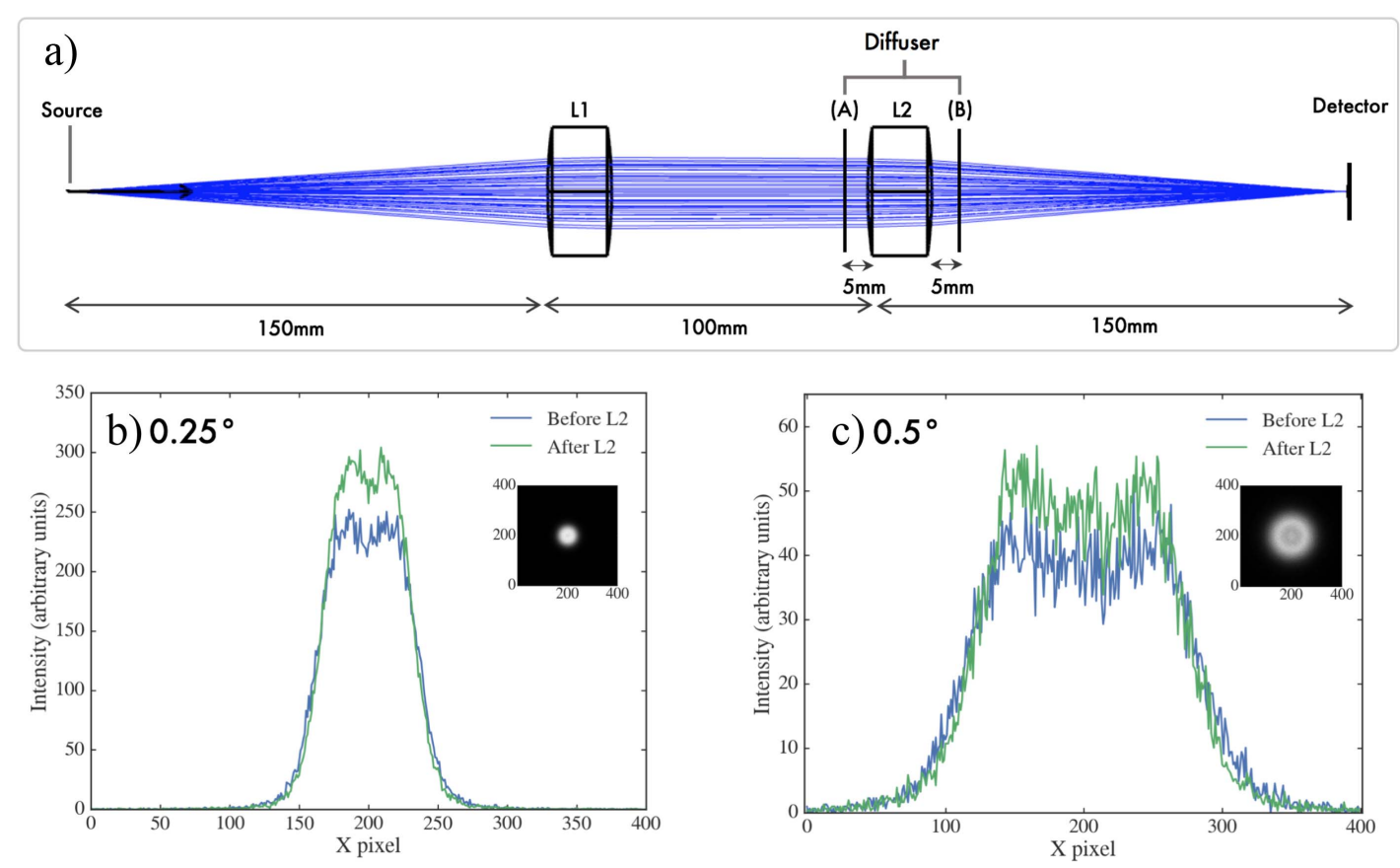

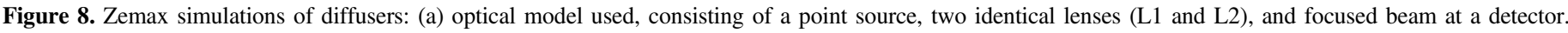

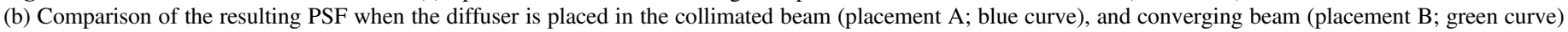
for a 0.25 off-the-shelf diffuser. (c) Same as (b), except for a 0.5 off-the-shelf diffuser.

incorporate a diffuser is in such a beam. However, some telescope systems have locations with collimated beams, where placing a diffuser would be more optimal. To compare the resulting PSF of a diffuser placed in a converging versus a collimated beam, we modeled off-the-shelf diffusers using Zemax Opticstudio in non-sequential mode. For these simulations, we used Bidirectional Scattering Distribution Function data files available from the RPC Photonics Web site measured for off-the-shelf diffusers illuminated with on-axis input beams.

Figure 8(a) shows an image of the optical model: an imaging system consisting of a point source emitting an $\mathrm{F} / 6.3$ beam at $550 \mathrm{~nm}$, a collimating lens (L1), and an identical lens (L2) to reimage the beam on a detector. Using this model, we studied the output at the detector by placing a diffuser in the collimated space before L2, and also in the converging beam after L2.

Figures 8(b) and (c) show the resulting horizontal cuts on the detector for an off-the-shelf diffuser with opening angles of 0.25 , and 0.5 , respectively. Also shown in the insets are the respective modeled PSFs. For both diffuser opening angles, we see that the PSF shape, size, and speckle structures (highintensity peaks in the image plane) are similar for a diffuser placed in the collimated and converging beams. However, we observe that the intensity for the diffuser in the converging beam is about $20 \%$ higher in both cases. This is due to two reasons. First, in the collimated beam, the diffuser is slightly farther away from the detector, broadening the resulting PSF. Second, with the diffuser in collimated space, the diffuser causes the incident rays on the L2 lens to be slightly diverging rather than collimated, moving the original focus position farther away. Although not specifically shown in Figure 8, this can be minimized by optimizing the focus of L2 after placing the diffuser in the collimated beam. With the diffuser in the collimated beam, an additional precaution will be to ensure that the now-diverging beam does not get clipped at the lens, i.e., that L2 has a large enough clear aperture to accommodate the larger beam footprint.

\subsubsection{Diffusers Can Be Rotated to Smooth Out Speckling}

The diffuser can be rotated during observation to smooth out the speckle pattern observed in diffused PSFs (Figure 9). Being a statistically varied pattern of engineered structures, illuminating different parts of the diffuser will result in slight variations in the resulting output PSF. This PSF variation can be averaged over time by moving the diffuser during an exposure. Therefore, by taking an exposure that is longer than the characteristic time of change in the residual PSF variation, the resulting PSF can be effectively smoothed out.

In practice, the least design-intensive path to move the diffuser in a beam is to rotate it along an axis parallel to the optical axis. We tested this in the lab: continuously rotating the diffuser at $\sim 1-2 \mathrm{~Hz}$ effectively smoothed the output PSF by allowing the diffuser to complete a few full rotations for exposure times of a few seconds. Figure 9 compares our lab measurements of rotated and non-rotated PSFs for two top-hat off-the-shelf diffusers from RPC Photonics with a 0.25 and 2.0 opening angle, respectively. Comparing the two nonrotating diffuser PSFs, we see that the amplitude of the speckles is larger for the 0.25 diffuser than for the 2.0 diffuser, or about $40 \%$ and $5 \%-10 \%$ of the total intensity, respectively. This results from the fact that it is easier to suppress speckling for larger opening angles.

Although not as good as allowing the diffuser to complete a few revolutions per exposure, we note that excellent PSF smoothing can already be achieved with only $\sim 180^{\circ}$ of rotation during an exposure. Therefore, considering that our shortest exposure times for ARCTIC for high-precision photometry on bright stars are on the order of 1-2 s-with most exposure times for high-precision photometry being longer than $10 \mathrm{~s}$ to maintain observing efficiency - the ARCTIC diffuser rotator was designed to produce a smooth rotational speed of at least $2-3 \mathrm{~Hz}$. 


\section{a) Not Rotating}
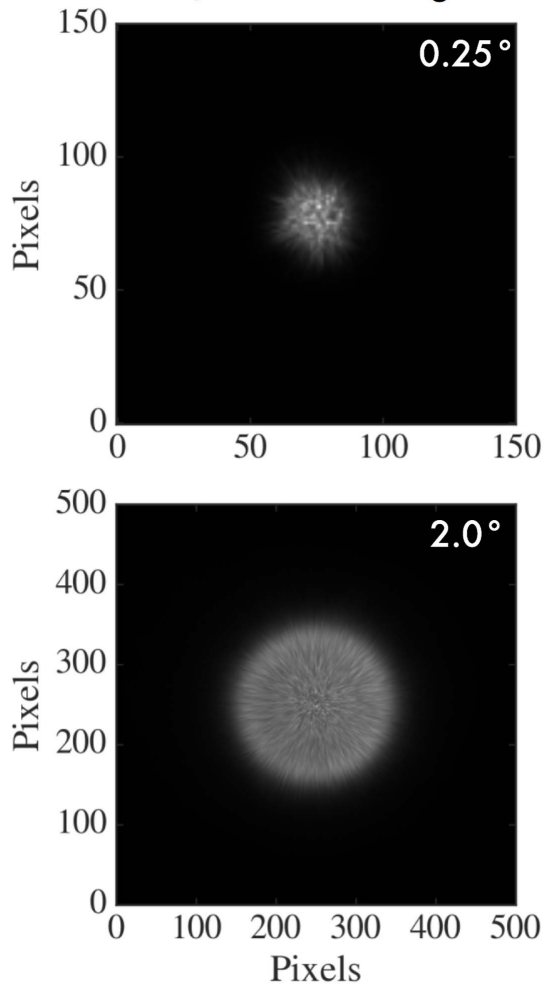

b) Rotating
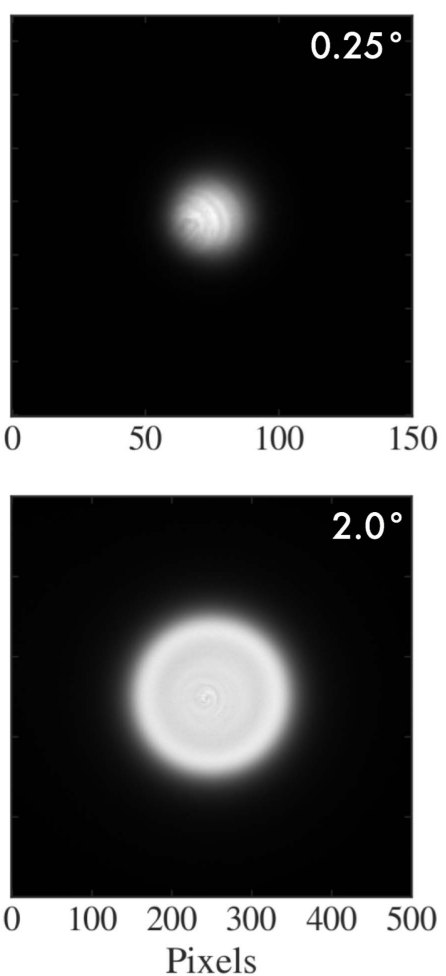

c) Horizontal cuts
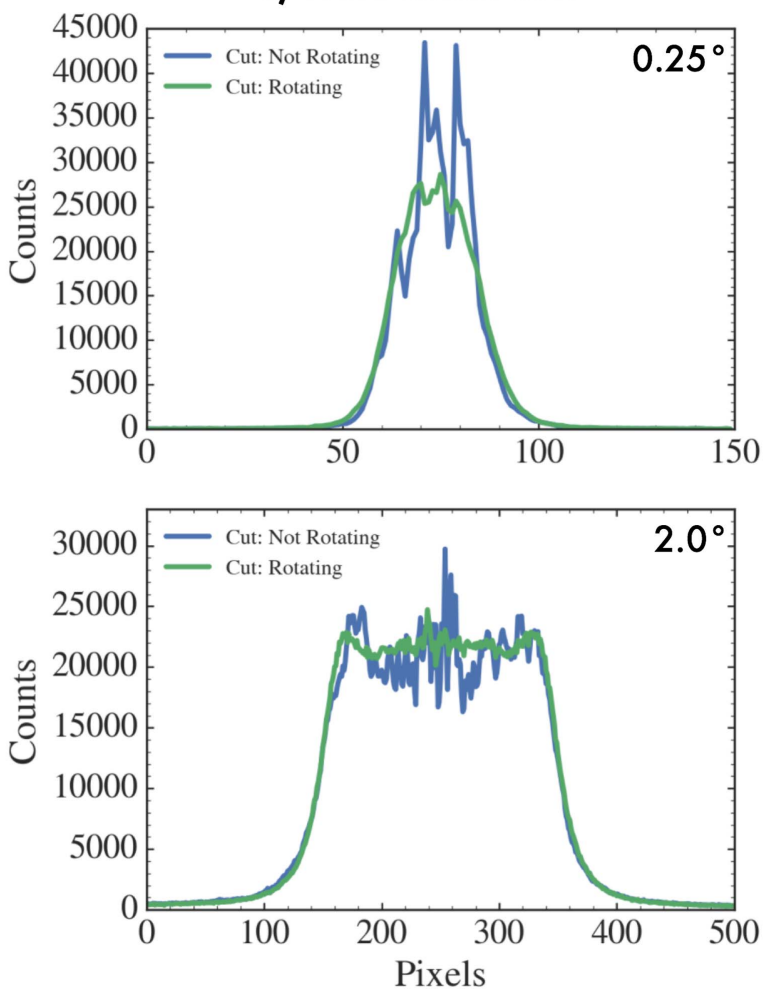

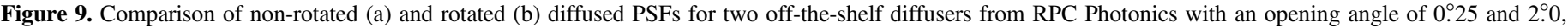
respectively. The panels in (c) show horizontal cuts through the center of the PSFs. The images in (a) and (b) have the same linear stretch.

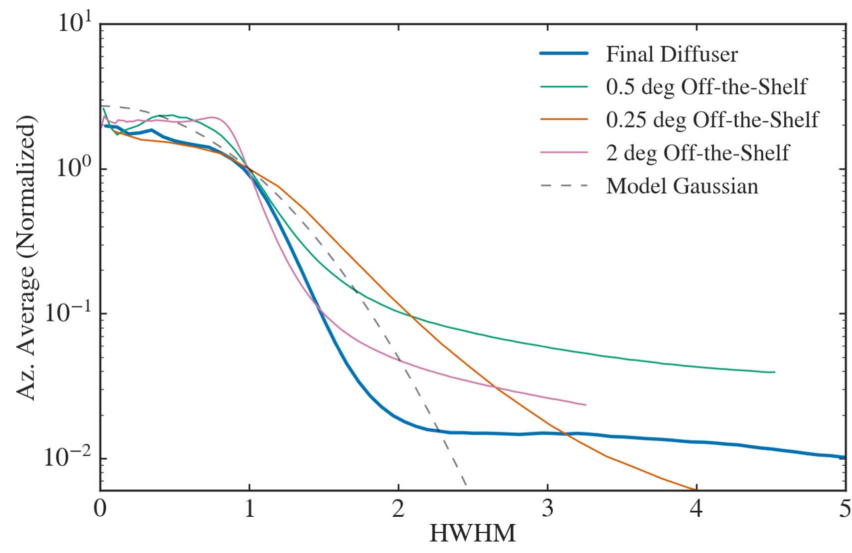

a)

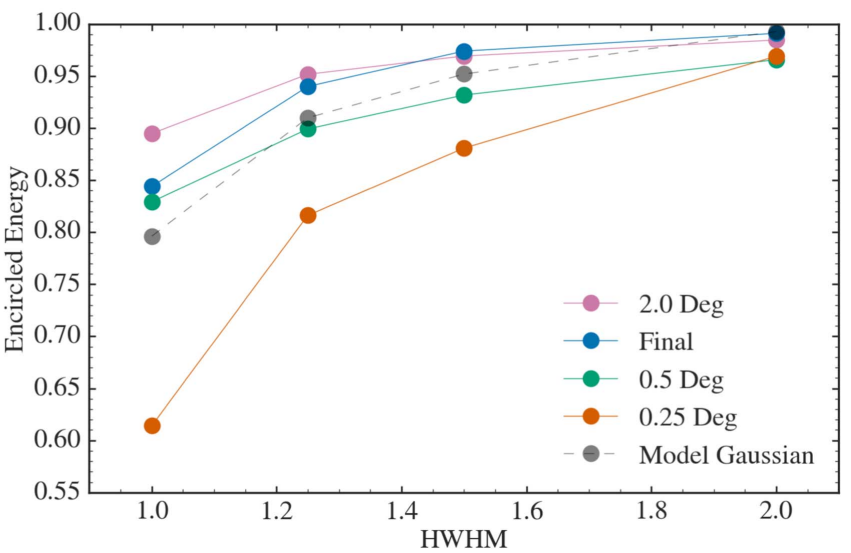

b)

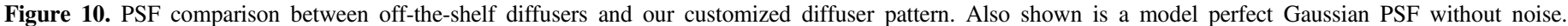

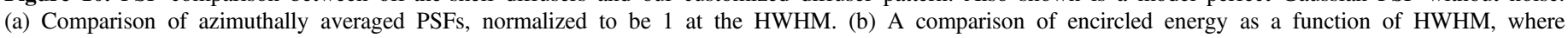
$\mathrm{EE}(3 \mathrm{HWHM})=1$. Our customized diffuser pattern is optimized to have a steep fall-off and a flat top.

Small residual ripples with amplitudes of $\sim 2 \%-10 \%$ of the total intensity are seen in the rotated PSFs in Figure 9. These ripples are concentric around the diffuser rotational axis. We see that for the 2.0 diffuser exposure (Figure 9) the rotational axis was more closely centered than in the 0.25 diffuser exposure. However, as shown in the horizontal cuts in Figure 9 (c), these ripples are small in comparison to the spikes before rotating.

We note that the diffusers in Figure 9 are marketed as general top-hat diffusers with a specific opening angle. As such, they are advertised to provide a top-hat PSF over a broad parameter space and were not specifically optimized for this test setup, i.e., to have a flat speckle pattern and steep wings. To find a diffuser more suited to our specific needs, we worked with RPC Photonics to design a custom top-hat diffuser that had better top-hat characteristics.

Figure 10(a) compares the azimuthally averaged PSF of our custom developed diffuser pattern to those of commercial offthe-shelf diffusers. To facilitate the comparison, Figure 10(a) shows azimuthally averaged PSFs normalized to be equal to unity at the HWHM of each PSF. We also show a model Gaussian with no background noise or background light. These data were taken at a fixed distance of $106 \mathrm{~mm}$ away from the detector. From Figure 10(a), we see that our customized 

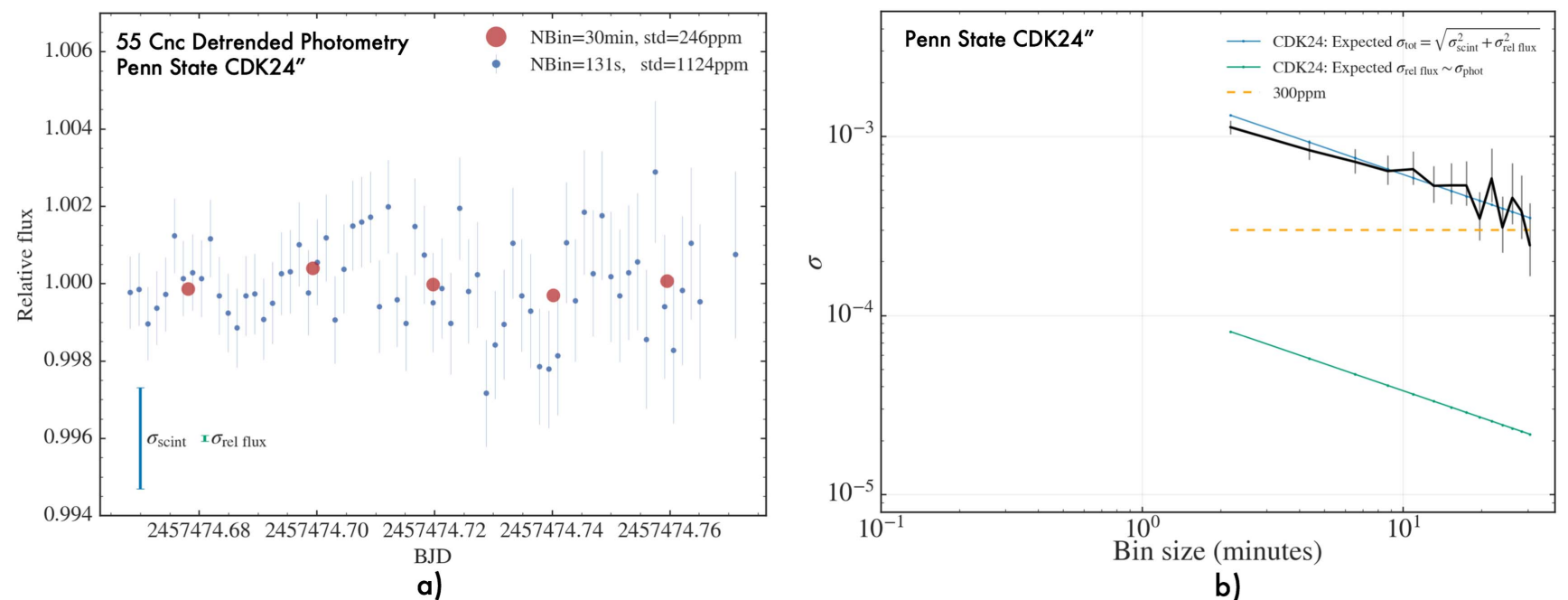

Figure 11. (a) Light curve of 55 Cnc (out of transit) as observed by the Penn State PlaneWave 24 inch telescope in the Johnson $I$ filter using a 2.0 diffuser. Using the diffuser allowed us to observe this bright star with a cadence of $131 \mathrm{~s}$, resulting in an unbinned precision of $1124 \mathrm{ppm}$. The photometric precision increases to $246_{-81}^{+176} \mathrm{ppm}$ in 30 minute bins. The error bars are the total noise error bars as calculated using Equation (7). Example scintillation and photon noise errors are shown in the bottom-left corner in blue and green, respectively. (b) Photometric precision as a function of bin size in minutes for our 55 Cnc observations (black). The green and blue curves show the expected calculated photon noise and total photometric noise, as calculated using Equations (2) and (7), respectively. We see that these observations are scintillation limited. The data used to create this figure are available.

diffuser pattern provides PSFs with steeper wings than produced by off-the-shelf diffusers. We see that for our customized diffuser, the PSF plateaus at a relatively constant level at $\sim 2 \mathrm{HWHM}$, indicating that most of the signal for this diffuser will be within $\sim 2$ HWHM. The other diffusers fall off less steeply and do not show evidence of a plateau in the range tested.

Figure 10(b) shows the encircled energy (EE; curve of growth) for the same set of diffusers tested. Due to the size of the detector used, we could only record up to $\sim 3 \mathrm{HWHM}$ of the PSF of the $2^{\circ} .0$ diffuser. Therefore, to compare the EEs, we set $\mathrm{EE}(3 \mathrm{HWHM})=1$ for all of the PSFs and compare how quickly the EE or signal strength grows as a function of HWHM. We see that the customized diffuser pattern is better than the model Gaussian and both off-the-shelf diffusers with a similar opening angle, in terms of steepness of fall-off and EE. In this comparison, the $2^{\circ} .0$ diffuser is observed to have better EE and a flatter top-hat, but still to have some residual power toward the higher angles (fall off of wings could be steeper). As mentioned above, it is easier to fabricate and design diffuser patterns producing closer to ideal top hats for diffusers with larger opening angles. However, for our astrophysical applications, we needed diffusers closer to or smaller then $\sim 0^{\circ} .4$ to achieve PSFs with an FWHM $\sim 10^{\prime}$ with ARCTIC to minimize the sky-background noise and source overlap effects.

\subsection{Diffusers on Sky}

\subsubsection{Optical Diffuser-assisted Photometry with Penn State's CDK 24}

Figure 11 shows a result from some of our early tests with off-the-shelf diffusers on a small telescope. Specifically, Figure 11 shows $2.5 \mathrm{hr}$ of out-of-transit observations of 55 Cnc using Penn State's CDK 24 telescope with a 2.0 off-theshelf diffuser from RPC Photonics. Using the diffuser allowed us to spread the PSF over a large number of pixels, 160 pixel FWHM, corresponding to $\sim 20,000$ pixels illuminated per target. Spreading out the PSF over so many pixels allowed us to increase our exposure time to $120 \mathrm{~s}$ and giving a final cadence of $131 \mathrm{~s}$ (11 s readout time), allowing us to achieve a duty cycle of $90 \%$ and gather $\sim 10^{8}$ electrons in the target and reference star apertures, respectively. The PSF remained stable throughout the observations. The resulting unbinned precision after detrending with airmass and the $x$ and $y$ pixel positions was 1124 ppm (see Figure 11).

Figure 11 shows how the 55 Cnc observations bin down with increasing bin sizes (black curve). We calculate the error bars on our photometric precision, using the code described in Cubillos et al. (2017), assuming that for the highest binning sizes, the rms scatter as a function of bin size follows an inverse-gamma distribution. The data bin down in a Gaussianlike manner, indicative of minimal residual systematic effects. In 30 minute bins, the data bin down shows a scatter of $246_{-81}^{+176} \mathrm{ppm}$. This precision is similar to the precision needed to successfully observe the transit of $55 \mathrm{Cnc}$ e (McArthur et al. 2004; Dawson \& Fabrycky 2010)—having a transit depth of 380 ppm (Winn et al. 2011) —in a single night of observations. Although we note that observing only one transit at this precision would result in a marginal detection of the transit, the transit could be more precisely be characterized by co-adding a few transit observations to further increase the precision. This demonstrates that the addition of such diffusers could significantly improve detection thresholds of transiting planets orbiting bright stars.

Furthermore, Figure 11 compares the relative photometric flux errors, $\sigma_{\text {rel flux }}$ (green curve), to the expected total photometric noise (including scintillation noise), $\sigma_{\text {tot }}=\sqrt{\sigma_{\text {rel flux }}^{2}+\sigma_{\text {scint }}^{2}}$ (blue curve), as calculated using Equations (2), and (7), respectively. For the airmass term in Equation (7), we assumed a fixed airmass equal to the mean airmass of the observations, amounting to $\chi \sim 1.4$, which matches well with the observed scatter. From Figure 11, we see that the total noise is much larger than the photometric noise by an order of magnitude $\left(\sigma_{\text {tot }} \gg \sigma_{\text {rel flux }}\right)$, where the total error is dominated by the scintillation noise. This is expected for a 24 


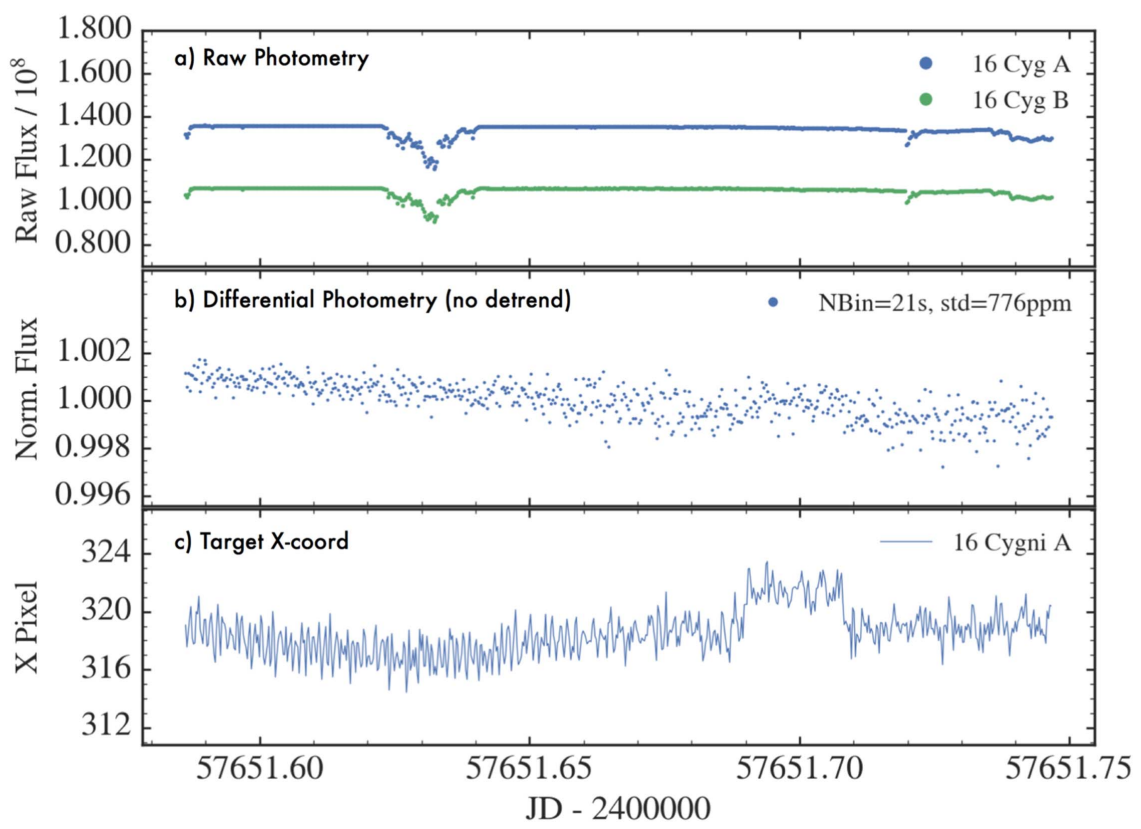

Figure 12. Demonstration of diffuser-assisted photometry in the optical: engineering-time observations of the bright binary star 16 Cyg A. (A) The raw photometry of 16 Cyg A and 16 Cyg B. Clouds are seen after $\sim 1 \mathrm{hr}$ from the start of the observations and also toward the end. (B) Normalized unbinned photometry of 16 Cyg A, without detrending. (C) $x$-pixel coordinate of the target star 16 Cyg A as a function of time.

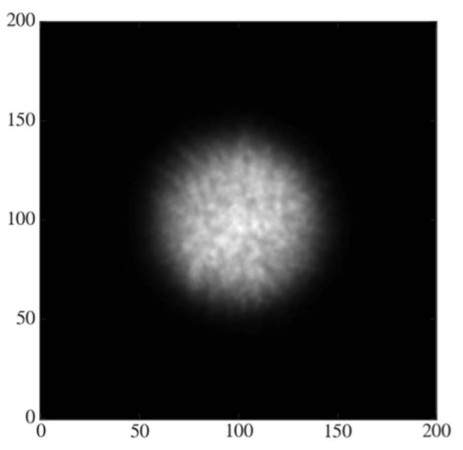

a)

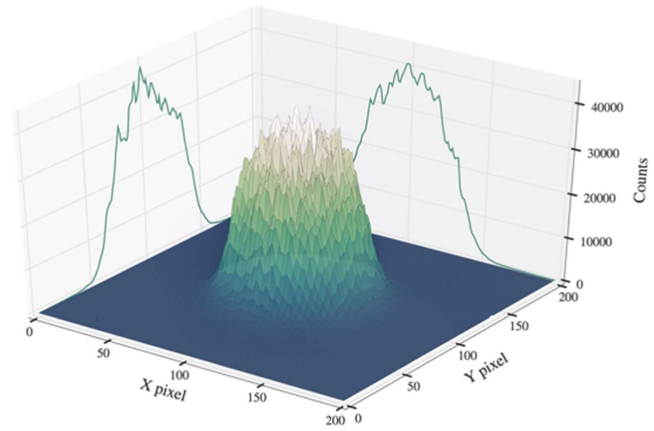

b)

Figure 13. 3D view of a diffuser image chosen at random from the 16 Cyg A photometry. The ARCTIC diffuser rotator was not working reliably during this observing run, and this image shows the PSF when the rotator was not rotating, showing the small-amplitude speckle pattern, which remained stable throughout the observations. An animation of this figure is available. The animation demonstrates that overall the PSF remains stable throughout the observations. The smoothing of the residual speckle pattern seen in the animation (e.g., toward the end of the video) is due to a combination of seeing effects and the rotator intermittently working. (An animation of this figure is available.)

inch $(0.6 \mathrm{~m})$ telescope observing such a bright star from Figure 2.

\subsubsection{Optical Diffuser-assisted Photometry with ARC $3.5 \mathrm{~m}$}

Photometric observations of 16 Cygni-To demonstrate the photometric precision capabilities of diffusers on sky, we show $4 \mathrm{hr}$ of differential photometry of 16 Cyg A, taken during our engineering run in 2016 September (see Figures 12-14). The unbinned raw photometry and unbinned differential photometry (without detrending) are shown in the top and middle panels in Figure 12, respectively. The unbinned undetrended precision is $776 \mathrm{ppm}$ for a $21 \mathrm{~s}$ cadence. From the top panel, we see that clouds appeared $\sim 1 \mathrm{hr}$ after the observations started (JD 0.63) and toward the end, but those transparency changes are effectively canceled out in the differential photometry in the middle panel. However, we do observe a slow downward linear trend in the differential photometry, along with a bump at around 0.70 JD with a clear correlation with the $x$ centroid coordinate of the target star (bottom panel).

The diffused PSF remained stable throughout the night. Figure 13 shows an image taken at random from this data set. Speckles are still seen in the diffused PSF, but they are smoothed to a certain extent by seeing variations and clouds passing by. An animation of the observations is available. During these observations, the rotator did not work reliably, as it was difficult to rotate the diffuser bearing due to friction. This caused the diffuser to sometimes rotate and sometimes get stuck. Therefore, although the rotator was formally on, the diffuser was only sporadically rotated during the observations presented. This is seen in the animation: the PSF speckle pattern is sometimes smoothed out. Regardless, the overall size and shape of the diffused PSF is not affected.

Figure 14(a) shows the final detrended photometry of 16 Cygni, detrended with a line, airmass, and the $x$ and $y$ centroid coordinates. The unbinned photometry (cadence: $21 \mathrm{~s}$ ) shows a 

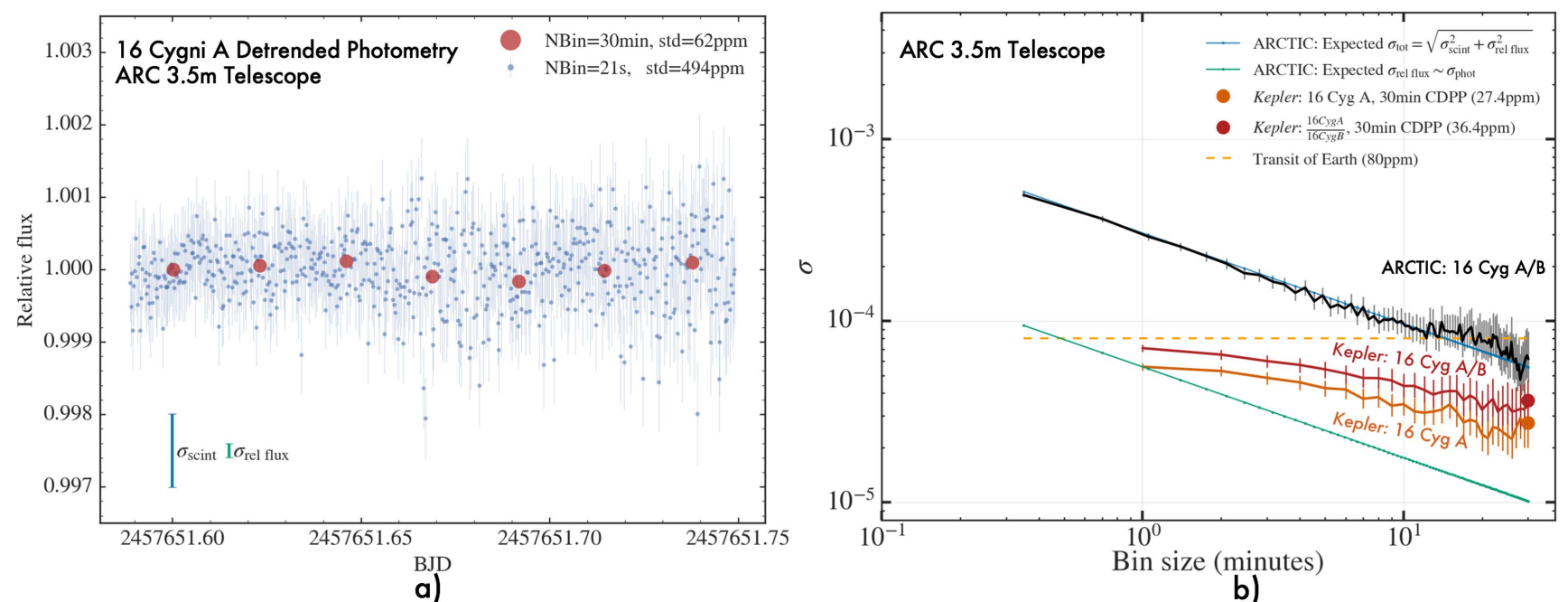

Figure 14. (a) Final detrended photometry of 16 Cyg A including scintillation errors, showing both unbinned (blue points) and in 30 min bins (red points). (b) Comparison of results with Kepler: photometric precision as a function of bin size in minutes, comparing the 16 Cyg A diffuser data to representative 4 hr shortcadence data of 16 Cyg A from Kepler (orange curve; see details in text). Additionally, as a head-to-head comparison with our ground-based differential photometry, we show the Kepler differential photometry for 16 Cygni A and B (red curve), which adds a $\sqrt{2}$ error when dividing the two light curves together. The bin size on the $x$-axis accounts for the full effective observing cadence (including both the exposure time and the dead time). The diffuser photometry reaches $62_{-16}^{+26}$ ppm precision in 30 minute bins, a factor of $\sim 2$ from Kepler. The data used to create this figure are available.

precision of $494 \mathrm{ppm}$, while in 30 minute bins, the $\mathrm{rms}$ precision bins down to $62_{-16}^{+26} \mathrm{ppm}$. The photometric error bars shown in Figure 14(a) are the total errors including scintillation, as calculated from Equation (7). The error bars increase toward the end due to the increasing airmass. Also shown is the calculated photometric noise error (including photon noise, dark, read, and sky-background noise), calculated using AstroImageJ from Equation (2) and a representative scintillation error calculated from Equation (6). The values for these errors in the unbinned photometry are $\sim 100 \mathrm{ppm}$ and $\sim 500 \mathrm{ppm}$, respectively, indicating that these observations are scintillation limited.

To study the effect of residual systematics, we plot the rms scatter of our 16 Cyg A observations as a function of increasing bin size (Figure 14(b)). Also overplotted are the relative photometric flux errors (green curve) as calculated with AstroImageJ (Equation (1)), including photon, dark, read, and sky-background noise, and the expected total photometric noise (including scintillation noise), $\sigma_{\text {tot }}=\sqrt{\sigma_{\text {rel flux }}^{2}+\sigma_{\text {scint }}^{2}}$ (blue curve), as calculated using Equations (5) and (7). For the airmass term in Equation (5), we assumed a fixed airmass equal to the mean airmass of the observations, amounting to $\chi=1.10$, which matches well with the observed scatter. We see that our data (black curve) largely bin down as white noise (blue curve).

In Figure 14(b), we also compare our precision to Kepler. We ran the $6.5 \mathrm{hr}$ combined differential photometric precision (CDPP) metric, as defined in Gilliland et al. (2011) for each of the individual quarters for 16 Cygni A in both long- and shortcadence mode, that were available from MAST. The resulting $6.5 \mathrm{hr}$ CDPP precision is consistently at the $7-9 \mathrm{ppm}$ level across different quarters, giving a $6.5 \mathrm{hr}$ CDPP precision of $>22 \mathrm{ppm}$, except for Quarter 6, which was known to have poor data quality due to the use of a non-optimized photometric aperture (Lund et al. 2014). Scaling this result to 30 minutes (i.e., $\sigma_{\mathrm{CDPP}, 30 \text { minutes }}=\sigma_{\mathrm{CDPP}, 6.5 \mathrm{hr}} \sqrt{6.5 \mathrm{hr} / 0.5 \mathrm{hr}}$ ) gives a precision ranging from 26 to $30 \mathrm{ppm}$ in 30 minute bins across the different quarters, with a median precision of $27.4 \mathrm{ppm}$. Calculating the standard deviation of the long-cadence data ( $\sim 30$ minute cadence), after throwing out $5 \sigma$ outliers and running the same two-day Savitsky-Golay high-pass filter as performed in Gilliland et al. (2010) and in the Everest 2.0 pipeline (Luger et al. 2016, 2017), we get a precision of $28-44 \mathrm{ppm}$ with a median value of $31.6 \mathrm{ppm}$, in 30 minute bins. This way of estimating the precision might be biased toward outliers, but it gives a precision estimate that is roughly consistent with the scaled CDPP precision. We choose to use the better of the two, or 27.4 ppm, as our Kepler precision comparison of $16 \mathrm{Cyg} \mathrm{A}$, and we plot this number in Figure 14(b). Additionally, in Figure 14(b), to graphically compare an equal length segment of Kepler photometry to our $4 \mathrm{hr}$ ground-based photometry, we plot a representative $4 \mathrm{hr}$ segment of the Kepler short-cadence data of 16 Cyg A. We say that this $4 \mathrm{hr}$ segment is "representative" of Kepler photometry on this star, as in 30 minute bins the precision of this $4 \mathrm{hr}$ segment is similar to the $27.4 \mathrm{ppm} 30$ minute CDPP precision value discussed above. Additionally, to perform a head-to-head comparison with our ground-based differential photometry to Kepler's differential photometry of 16 Cyg A, we calculated the same 30 minute CDPP metric for Quarter 7 on the differential16 Cyg A short-cadence Kepler light curve, using $16 \mathrm{Cyg} \mathrm{B}$ as a reference star. In doing this, the resulting 30 minute CDPP metric for the 16 Cyg Quarter 7 differential light curve degraded to $36.3 \mathrm{ppm}$, effectively adding a factor of $\sqrt{2}$ to the photometric noise. By this metric, our ground-based precision of 16 Cyg A of $62_{-16}^{+26} \mathrm{ppm}$ in 30 minute bins is a factor of $<2$ from Kepler's differential precision ( $37.4 \mathrm{ppm})$, but a factor of $\sim 2$ in the non-differential photometry case $(27.4 \mathrm{ppm})$. We show the exact $4 \mathrm{hr}$ segments of Kepler photometry of 16 Cyg A and B used in Figure 18(a) in Appendix A. 

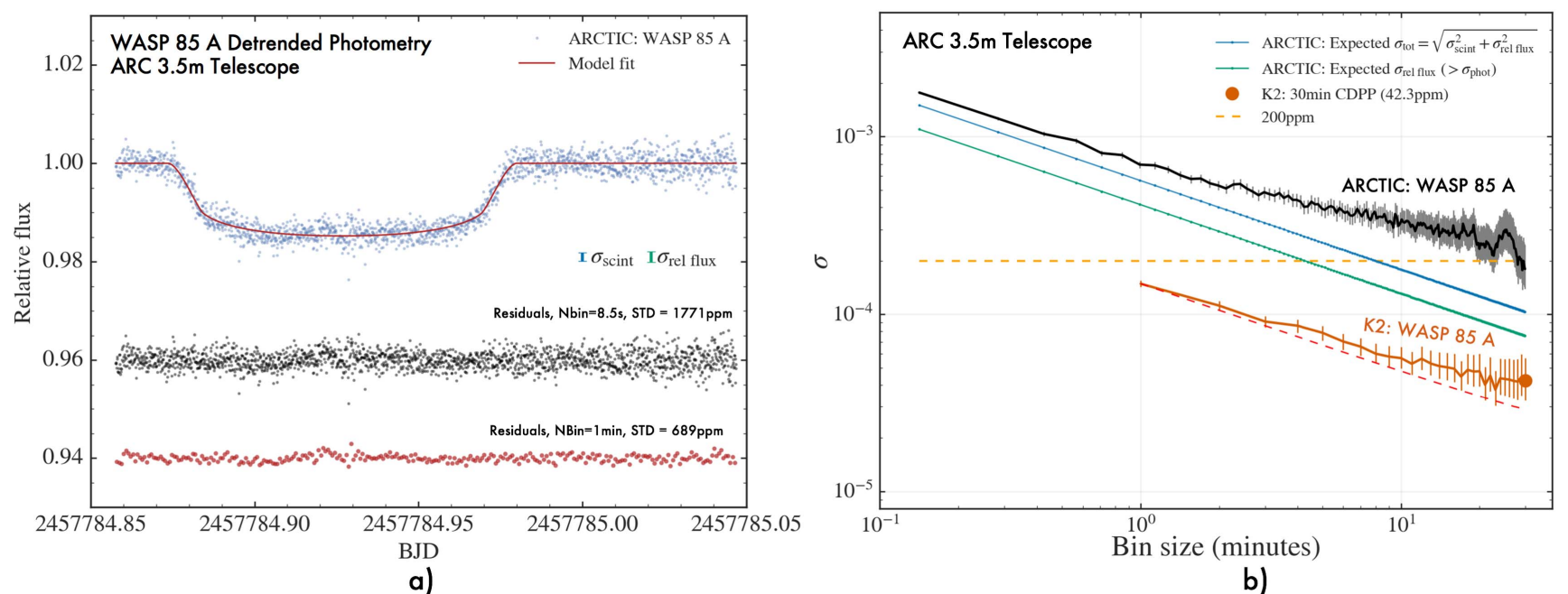

b)

Figure 15. (a) Transit of WASP-85 A b observed with the diffuser on ARCTIC, along with a best-fit model, and residuals offset for clarity. The unbinned precision (black points) is $1771 \mathrm{ppm}$, while binning to 1 minute, gives a precision of $689 \mathrm{ppm}$ (red points). We attribute the "bump" around the middle of the transit to the planet crossing a starspot. (b) Photometric precision comparison with K2: photometric precision as a function of bin size in minutes for our $4.5 \mathrm{hr}$ of ground-based diffuserassisted transit observations of WASP-85 A b (black curve) compared to a representative $4.5 \mathrm{hr} K 2$ short-cadence photometry of the same star out of transit (orange curve; see the text and Figure 18 for details). Additionally shown is the relative photometric error, $\sigma_{\text {rel flux }}$ (green curve) for our diffuser observations, along with the total expected error $\sigma_{\text {tot }}$ (blue curve). The diffuser-assisted observations reach $180_{-41}^{+66} \mathrm{ppm}$ in 30 minutes. The data used to create this figure are available.

Transit of WASP-85 A $b$-Figure 15(a) shows the transit of WASP-85 A b as observed with ARCTIC, along with our bestfit transit model after detrending the light curve with the $x$ and $y$ pixel coordinates, airmass, and a line. The unbinned residuals are shown offset for clarity, showing a photometric precision of $1771 \mathrm{ppm}$. Also shown are the residuals binned to 1 minute bins, with a precision of $689 \mathrm{ppm}$. Additionally shown in the plot are representative scintillation and photon noise error bars.

We observe a small brightening_or "bump" - in the middle of the transit. We speculate that this is caused by the planet crossing a starspot, as there is no clear correlation with other observational parameters, suggesting the bump is of astrophysical origin. This is a likely scenario, as WASP-85 A has been observed to have repeated starspot crossing events through detailed analysis of $K 2$ data (Močnik et al. 2016). If the bump in Figure 15(a) is indeed a starspot crossing, this marks the first ground-based detection of a starspot crossing event for this target.

Figure 15(b) shows how the rms scatter of the WASP-85 A b residuals change with increasing bin size. Also shown are the expected $\sigma_{\text {rel flux }}$ (green curve) and total errors $\sigma_{\text {tot }}=\sqrt{\sigma_{\text {rel flux }}^{2}+\sigma_{\text {scint }}^{2}}$ (blue curve), as calculated using Equations (2) and (7), respectively. The scintillation noise was calculated using Equation (6) assuming three reference stars and using the mean airmass of the observations of 1.3. Different from our 16 Cygni observations, where $\sigma_{\text {rel flux }} \ll \sigma_{\text {scint }}$, these observations are in the regime where $\sigma_{\text {rel flux }} \sim \sigma_{\text {scint }}$. We do observe that the unbinned precision (1771 ppm) we obtained is slightly higher than the expected one (1500 ppm). This might be attributed to some degree to the uncertainty in the estimation of the scintillation noise, which has a strong dependence on the variable wind profile in the upper atmosphere (Osborn et al. 2015). An additional factor helping explain this discrepancy is that the three reference stars are all much fainter than the target star (having fluxes of 3\%, $10 \%$, and $3 \%$ of the target star, respectively), lowering their
$\mathrm{S} / \mathrm{N}$ when correcting for atmospheric transparency fluctuations. Lastly, some of the correlated noise behavior toward higher bin sizes in Figure 15(b) might be explained by astrophysical noise, including the candidate starspot crossing event.

Similar to Figure 14, we compare our achieved precision on WASP-85 A with the 30 minute CDPP precision achieved by $K 2$ in Figure 15(b). The $K 2$ short-cadence photometry of WASP-85 A from Campaign 1 was retrieved and detrended with the Everest 2.0 pipeline (Luger et al. 2016, 2017). The $6.5 \mathrm{hr}$ CDPP precision of $K 2$ as calculated by Everest is $11.7 \mathrm{ppm}$, and using a similar scaling to the discussion above to calculate the 30 minute CDPP precision (i.e., $\sigma_{\mathrm{CDPP}, 30 \text { minutes }}=$ $\sigma_{\mathrm{CDPP}, 6.5 \mathrm{hr}} \sqrt{6.5 \mathrm{hr} / 0.5 \mathrm{hr}}$ ) yields a 30 minute CDPP of $42.3 \mathrm{ppm}$. Similarly to the discussion above for $16 \mathrm{Cyg}$ A in Figure 14(b), in Figure 15(b) we plot the $\sigma$-versus-bin-size curve for a representative $4.5 \mathrm{hr}$ segment of $K 2$ short-cadence data, the same length as our observations with ARCTIC. The specific segment is shown in Figure 18(b) in Appendix A. We see that on this star, our 30 minute ground-based precision $\left(180_{-41}^{+66} \mathrm{ppm}\right)$ is a factor of $\sim 4$ of the $K 2$ observations on this star (42.3 ppm).

We note that our precision of $180_{-41}^{+66} \mathrm{ppm}$ in 30 minute bins for our WASP-85 A b transit observations is worse than the precision for our $16 \mathrm{Cyg}$ A observations. This is due to a few reasons. First, $16 \mathrm{Cyg}$ A and B are both brighter than WASP$85 \mathrm{~A}$ and the available reference stars in the WASP-85 A field. This allowed us to suppress the photon noise better for the 16 Cyg A observations, while the WASP-85 A b transit observations are in the regime where the photon noise and scintillation noise are similar. Additionally, $16 \mathrm{Cyg}$ A and B are spectrally well matched, reducing the effects from secondary extinction. Second, for the 16 Cyg A observations, we used a filter (Semrock 857/30) with little-to-no water absorption in the red-optical, minimizing systematics due to molecular absorption and extinction. Furthermore, astrophysical systematics for WASP-85 A, in particular from our 


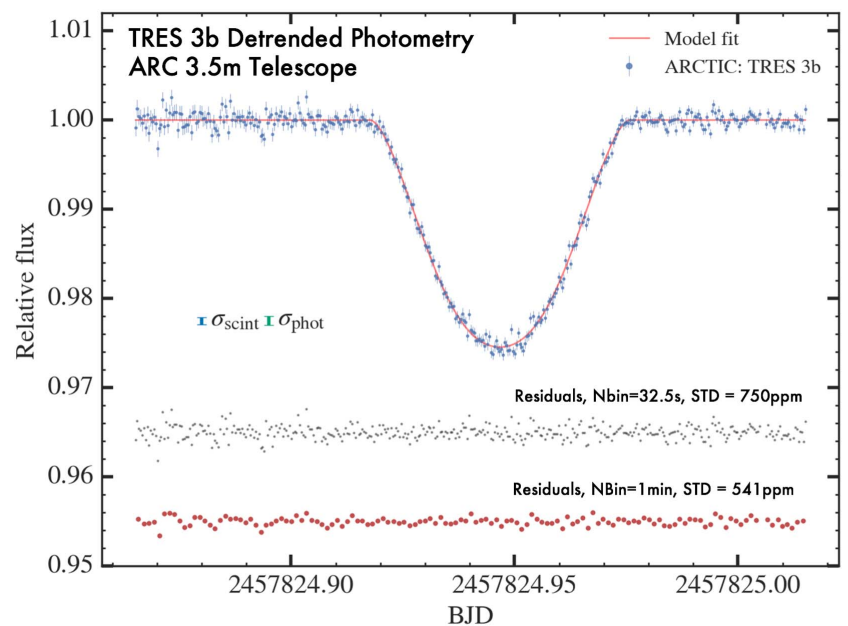

a)

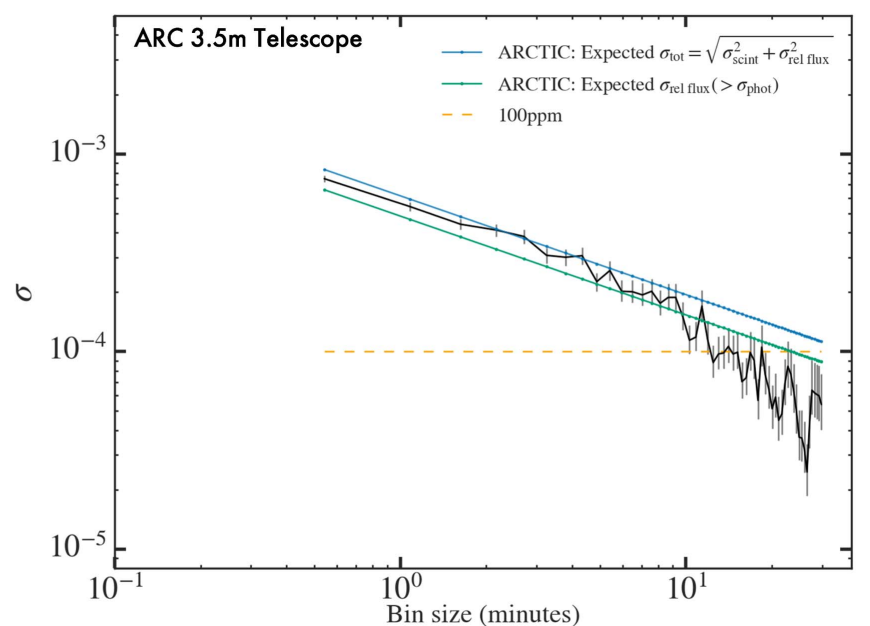

b)

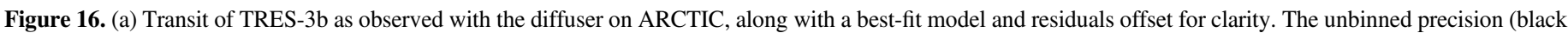

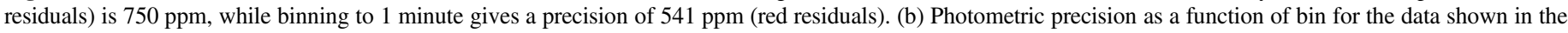

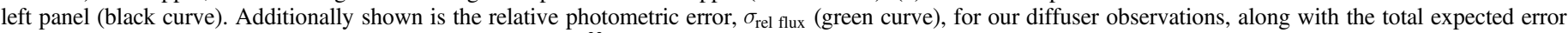

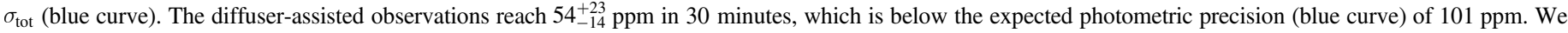
conservatively say that we reach a precision of $101 \mathrm{ppm}$ in 30 minute bins. The data used to create this figure are available.

candidate starspot crossing event, further add to the systematic noise floor for those observations. Still, even though the precision level achieved for our WASP-85 A transit observations is worse than for our 16 Cygni observations, the precision of $180_{-41}^{+66} \mathrm{ppm}$ in 30 minutes demonstrates that diffusers are enabling precision observations across a wide magnitude range, even for targets with overlapping PSFs.

Transit of TRES-3b-Figure 16(a) shows our in-transit observations of TRES-3b as observed by ARCTIC with a diffuser, along with our best-fit transit model after detrending the light curve with $x$ and $y$ pixel centroids and airmass. We did not detrend with a straight line as we did for the other observations, as it did not yield a significant improvement in the rms scatter.

Figure 16(b) shows how the rms scatter of the TRES-3b residuals change with increasing bin size, similar to Figures 11(b), 14(b), and 15(b). Furthermore, Figure 16(b) compares the relative photometric flux errors, $\sigma_{\text {rel flux }}$ (green curve), to the expected total photometric noise (including scintillation noise), $\sigma_{\text {tot }}=\sqrt{\sigma_{\text {rel flux }}^{2}+\sigma_{\text {scint }}^{2}}$ (blue curve), as calculated using Equations (2) and (7), respectively. For the scintillation term in Figure 14, we assumed a fixed airmass equal to the mean airmass of the observations $(\chi=1.20)$ and 10 reference stars. For the smallest bins, we see that the scatter bins down roughly as white noise. However, at the largest bins, we see a dip in the precision below the expected Gaussian behavior at bin sizes $>10$ minutes: the data bin down to $54_{-14}^{+23} \mathrm{ppm}$ in 30 minute bins (black curve), slightly below the expected photometric precision of $101 \mathrm{ppm}$ (blue curve). The drop in Figure 16(b) is somewhat below the expected photometric noise (green curve in Figure 16(b)), but is overall largely within the $1 \sigma$ or $2 \sigma$ error bars. Similar excursions below the expected Gaussian behavior at large bin sizes have been reported in the literature (e.g., Blecic et al. 2013; Cubillos et al. 2013), and Cubillos et al. (2017) demonstrate that those fluctuations are not statistically significant after taking into account the increasingly skewed inverse-Gamma distribution of the bins at large bins sizes. Therefore, we argue that a precision much below the expected photometric noise (blue curve) is likely an overestimate of the actual precision, and we thus conservatively say that our achieved precision in 30 minutes for these observations equals the expected photometric precision of $101 \mathrm{ppm}$. We discuss this further in Section 6, where we compare our precision with other values reported in the literature.

\subsection{NIR Diffuser-assisted Photometry}

Figure 17 shows our initial test results with the diffuser in the NIR, showing the reduced light curve of the target star 2MASS J04230271+5740319 after normalizing with the 10 reference stars used and the median flux. The original scatter of the light curve is 1232 ppm before any binning.

Figure 17(b) shows how the scatter of the binned light curve changes with increasing bin size, where we also show the expected photon noise (excluding background noise) for these observations in orange, along with the scintillation noise as calculated using Equation (7), added in quadrature to the photon noise. We see that the scatter of the binned light curve follows largely the expected binning of Gaussian white noise, and similar to the TRES-3 observations above (Figure 16(b)), we observe an excursion below the expected photon noise (orange curve) at the highest bin sizes. Similar to the TRES-3 observations, we attribute this behavior to the small number of bins at the higher bin sizes. The data (black curve) bins down to $137_{-36}^{+64} \mathrm{ppm}$ in 30 minute bins, which is above the expected total noise of $\sigma_{\text {tot }} \sim 100 \mathrm{ppm}$. These precision levels of $137_{-36}^{+64} \mathrm{ppm}$ in 30 minute bins are among the best broadband photometric precisions achieved in the NIR from the ground.

For these observations, the expected scintillation is small due to the large aperture of the telescope, the large number of reference stars used, along with the low airmasses of the observations. We note, however, the important caveat that the total error, $\sigma_{\text {tot }}$ (blue curve), does not include a term from the background noise, as the previous figures have, which will 


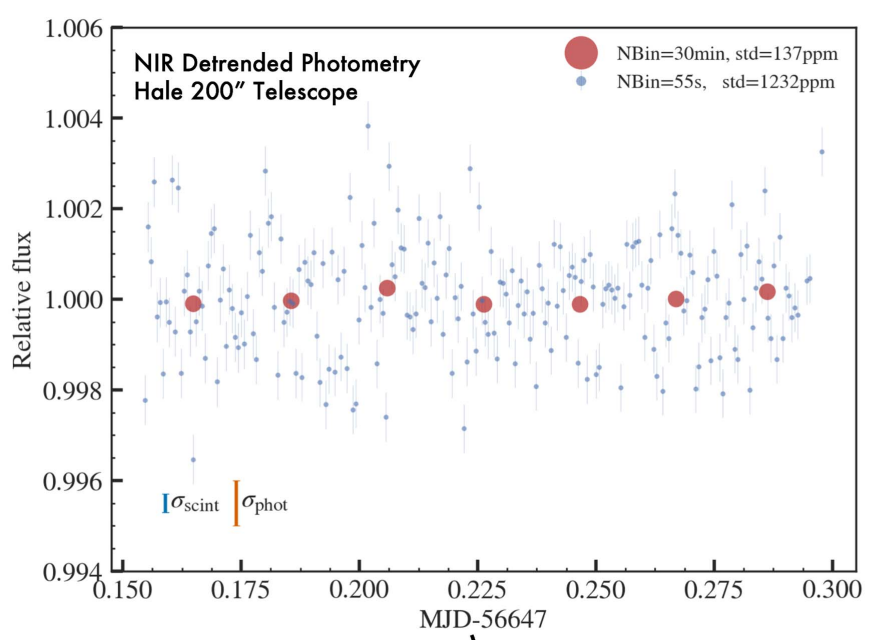

a)

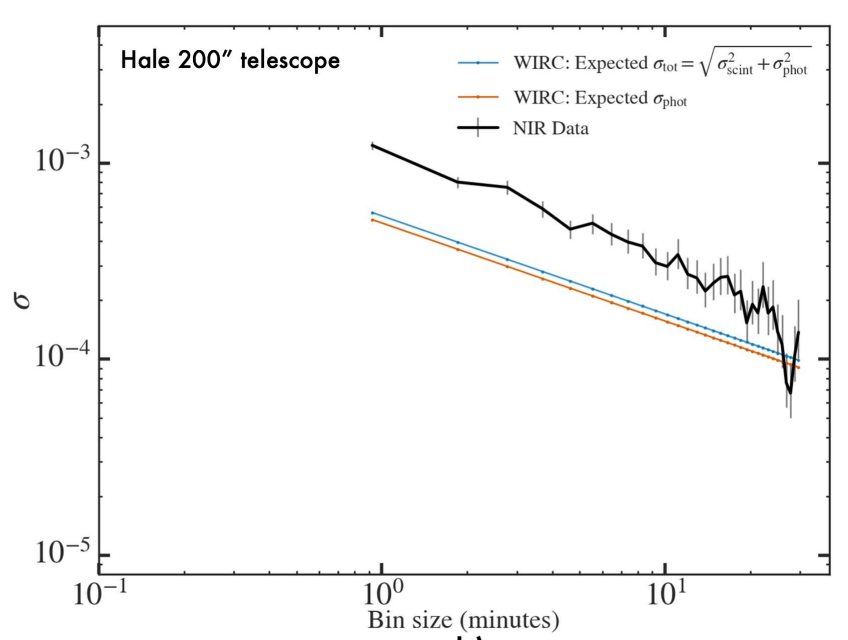

b)

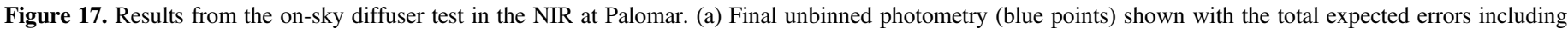

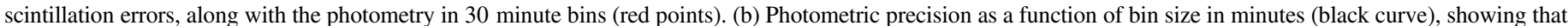

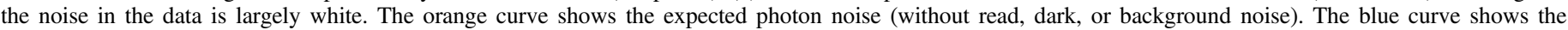

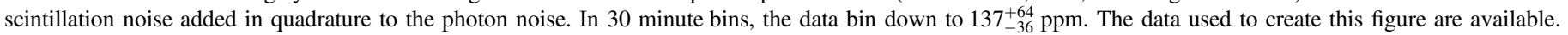

be particularly important in the NIR. We did not calculate the background noise term here, as these observations used a dedicated dithered sky-background master frame subtracted from the raw images to create the final science frames, different from our observations in the optical.

\section{Transit MCMC Fitting}

To check that our planet parameters agree with the values reported in the literature, we performed Markov Chain Monte Carlo (MCMC) fitting to the light curves of WASP-85 A b and TRES-3b. We performed our MCMC fitting following a $\chi^{2}$-minimization modeling approach similar to the EXOFAST code as described in Eastman et al. (2013). Different from EXOFAST, our code is in Python, which utilizes the BATMAN Python package (Kreidberg 2015), which uses the Mandel \& Agol (2002) transit model formalism. Similar to EXOFAST, we first use an amoeba minimization algorithm to find a good $\chi^{2}$ minimum in the parameter space describing the transit. We use those parameter values as starting points for our MCMC chains, initializing 30 walkers for the emcee affine-invariant ensemble sampler (Foreman-Mackey et al. 2013), in a small Gaussian ball centered around the amoeba best-fit values.

For our MCMC chains, we used five jump parameters describing the planet transit, $T_{0}, \log (P), \cos (i), R_{p} / R_{*}$, and $\log \left(a / R_{*}\right)$, along with one parameter for the out-of-transit baseline flux. Following the modeling efforts of the discovery papers for WASP-85 A b (Brown et al. 2014) and TRES-3b (O'Donovan et al. 2007), we fix the eccentricity and the argument of periastron to be equal to 0 . To account for systematic correlated noise in our data, we include up to four additional detrending coefficients in the $\chi^{2}$-minimization including the normalized airmass, $x$ and $y$ centroid pixel coordinates, and a straight line through the data. For the WASP-85 A b, we use all four detrending parameters, but for TRES-3b, we use the first three detrending parameters, as detrending with a line did not improve the quality of the fit.
Our priors are summarized in Table 4. For WASP-85 A b, we adopt Gaussian priors on the planet parameters from Brown et al. (2014), which we propagated accordingly using standard error propagation to describe our modified MCMC jump parameters (Table 4). For TRES-3b, we adopt Gaussian priors on the stellar and planet parameters from Sozzetti et al. (2009), but we adopt our priors on the transit ephemeris from the more recent work of Jiang et al. (2013). For both transits, we kept the limb-darkening parameters fixed, calculating their values using a quadratic limb-darkening law from Claret \& Bloemen (2011) for the respective bandpasses using the EXOFAST limbdarkening Web applet, ${ }^{26}$ which uses the host star $\log g, T_{\text {eff }}$, and $[\mathrm{Fe} / \mathrm{H}]$ values. To account for the light-curve flux dilution from the WASP-85 B binary companion in the WASP-85 A b light curve, we follow the approach described in Brown et al. (2014), adopting a fixed dilution factor for third light in our observing band of $L_{3, \mathrm{SDSS}} \mathrm{i}^{\prime}=0.5$ to the $R_{p} / R_{*}$ ratio. For the detrending parameters, we chose Gaussian priors on the best-fit values found by the initial amoeba $\chi^{2}$-minimization.

After initializing each MCMC walker in emcee, we ran each walker for 5000 steps and threw the first 1000 steps out as burn-in. Two-dimensional corner plots of the resulting chains are shown in Figures 19 and 20 in Appendix B. Furthermore, to check if the chains were ready for inference, we followed the suggestion by Ford (2006) to check the Gelman-Rubin statistic, $\hat{R_{\nu}}$, which was within $5 \%$ of unity for all of the parameters. The mean acceptance fractions in the sampling of the MCMC chains were $\sim 43 \%$ and $\sim 4 \%$ for the WASP-85 A $\mathrm{b}$ and TRES-3b light curves, respectively. The results from our MCMC fits are listed in Table 5, showing the median best-fit planet parameters, along with $68 \%$ confidence intervals.

For WASP-85 A b, our overall planet parameters agree well with the values reported in Brown et al. (2014), including our best-fit planet radius of $1.515_{-0.043}^{+0.044} R_{J}$. However, both our value and the value reported by Brown et al. (2014; $\left.R_{p}=1.48 \pm 0.03 R_{J}\right)$ are somewhat higher than the value reported by Močnik et al. (2016). We speculate that this

\footnotetext{
${ }^{26}$ http://astroutils.astronomy.ohio-state.edu/exofast/limbdark.shtml
} 
Table 4

Summary of Priors for TRES-3b and WASP-85 A b

\begin{tabular}{|c|c|c|c|}
\hline Parameter & Description & WASP-85 A b & TRES-3b \\
\hline \multicolumn{4}{|c|}{ Stellar Parameters } \\
\hline$R_{*}\left(R_{\odot}\right)$ & Stellar radius & $0.935 \pm 0.023$ & $0.829 \pm 0.022$ \\
\hline$T_{\text {eff }}(\mathrm{K})$ & Stellar effective temperature & $5685 \pm 65$ & $5650 \pm 75$ \\
\hline$[\mathrm{Fe} / \mathrm{H}]$ & Stellar metallicity & 0.08 & -0.19 \\
\hline $\log (g)$ & Stellar gravity & 4.519 & 4.568 \\
\hline \multicolumn{4}{|c|}{ MCMC Transit Jump Parameters } \\
\hline$T_{C}\left(\mathrm{BJD}_{\mathrm{TDB}}\right)$ & Transit midpoint & $2457784.927 \pm 0.00020$ & $2457824.94589 \pm 0.00006$ \\
\hline $\log (P)$ (days) & Orbital period & $0.424175367 \pm 0.00000007$ & $0.116005357 \pm 0.00000005$ \\
\hline $\cos (i)$ & Transit inclination & $0.0054 \pm 0.0019$ & $0.1418 \pm 0.0028$ \\
\hline$R_{p} / R_{*}$ & Radius ratio & $0.1358 \pm 0.0046$ & $0.1653 \pm 0.0063$ \\
\hline $\log \left(a / R_{*}\right)$ & Normalized orbital radius & $0.951 \pm 0.011$ & $0.7724 \pm 0.0076$ \\
\hline \multicolumn{4}{|c|}{ MCMC Fixed Parameters } \\
\hline$\sqrt{e} \cos (\omega)$ & Eccentricity \& argument of periastron & 0 (adopted) & 0 (adopted) \\
\hline$\sqrt{e} \sin (\omega)$ & Eccentricity \& argument of periastron & 0 (adopted) & 0 (adopted) \\
\hline$u_{1}$ & Linear limb-darkening coefficient & 0.4122 & 0.3145 \\
\hline$u_{2}$ & Quadratic limb-darkening coefficient & 0.2680 & 0.2704 \\
\hline$L_{3, \mathrm{SDSS} \mathrm{r}^{\prime}}$ & Dilution ratio & 0.50 & $\cdots$ \\
\hline
\end{tabular}

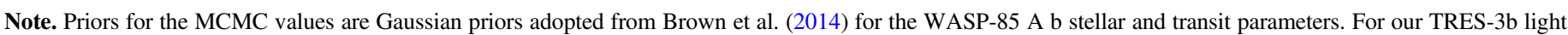
curve, we adopt priors from Sozzetti et al. (2009) on the stellar parameters and more recent parameters from Jiang et al. (2013) on the transit ephemeris.

discrepancy is due to systematics arising from observing the target in different filters-with the work presented here and in Brown et al. (2014) done in red-optical filters, and the work by Močnik et al. (2016) in the blue Kepler bandpass-due to contaminating light from the WASP-85 B binary companion. Furthermore, we note that our median value for the orbital inclination of $88.89_{-1.1}^{+0.77}$ is somewhat lower than the inclination reported by Brown et al. $\left(2014 ; 89^{\circ} .72_{-0.24}^{+0.18}\right)$ and Močnik et al. $\left(2016 ; 89^{\circ} 69_{-0.03}^{+0.11}\right)$. Looking at our $\cos (i)$ posteriors in Figure 19 in Appendix B.1, we see that our $\cos (i)$ inclination distribution does not approximate a Gaussian profile. We attribute this to the $\cos (i)$ inclination distribution being a positive-definite parameter (as we constrain $i<90^{\circ}$ ), causing the median of the $\cos (i)$ distribution to be biased toward positive values, although the mode of the distribution is close to or consistent with 0, similar to the Lucy \& Sweeney (1971) eccentricity bias.

For TRES-3b, our overall planet parameters agree well with the values reported by Sozzetti et al. (2009). We note that our best-fit planet radius of $1.377_{-0.036}^{+0.037} R_{J}$ is slightly higher than the value of $1.336_{-0.037}^{+0.031} R_{J}$ in Sozzetti et al. (2009). Acknowledging that $R_{p} / R_{*}$ can be sensitive to the exact detrending parameters chosen for ground-based observations (e.g., Villanueva et al. 2016), and as our value is within $2 \sigma$ from the values reported by Sozzetti et al. (2009), we conclude that our value is consistent with their result. Assuming the ephemeris from Jiang et al. (2013) for TRES-3b, our transit midpoint of $T_{C}=2457824.946728_{-0.000008}^{+0.00009}$ differs by only 1 minute from the expected linear ephemeris, demonstrating that our transit ephemeris shows no evidence for significant transit timing variations.

\section{Discussion}

\subsection{A Comparison with Other High Precision Photometry}

We compare our photometric precision to some of the highest ground-based precisions reported in the literature in Table 6, in the optical and NIR. The methods used in Table 6 to achieve these photometric precisions include telescope defocusing (Gilliland et al. 1993; Croll et al. 2011; Kundurthy et al. 2013; Tregloan-Reed \& Southworth 2013; Zhao et al. 2014; Fukui et al. 2016), using orthogonal-transfer CCDs (Johnson et al. 2009), tunable filter narrow-band spectrophotometry (Colón et al. 2012), or diffusers (this work). Our goal here is to survey some of the best published photometric precision values from the ground and to show that the first diffuser-assisted photometric precisions are already paralleling the best precisions in the literature.

The most direct way to compare the photometric precision levels achieved across these studies is to compare the precision per unit time. Another comparison metric is to compare how close the achieved precision is to the expected photon and scintillation limit, as we have done in Figures 11, and 14-17. However, this is often challenging to do for other efforts in the literature without knowledge of the telescope throughput and/ or with the image frames in hand, as the scintillation and photon noise errors are often not reported. Due to these uncertainties, we restrict our comparison in Table 6 to comparing reported photometric precision levels in the respective papers per unit time, and only compare them to the photon noise limit when explicitly stated in the paper.

For this comparison, we choose to use two metrics. First, we compare the precision levels per one minute effective cadence, $\sigma_{1 \text { minutes, }}$, a regime where the correlated noise in practice is a minimal fraction of the overall photometric noise budget. To acquire the $\sigma_{1 \text { minutes }}$ values, we either adapt it directly as mentioned in the paper, or if the precision in 1 minute is not specifically reported, we calculate a scaled value from the unbinned precision assuming pure Gaussian white noise behavior, i.e., using $\sigma_{1 \text { minutes }}=\sigma_{\text {unbinned }} / \sqrt{60 \mathrm{~s} / t_{\text {cadence }}}$, where $t_{\text {cadence }}$ is the effective cadence of the observations (time between successive exposures). Second, we also choose to compare the reported precision levels at 30 minute bins as $\sigma_{30 \text { minutes. }}$ This is the binning regime where the correlated noise can be a significant fraction of the overall error. The level of 


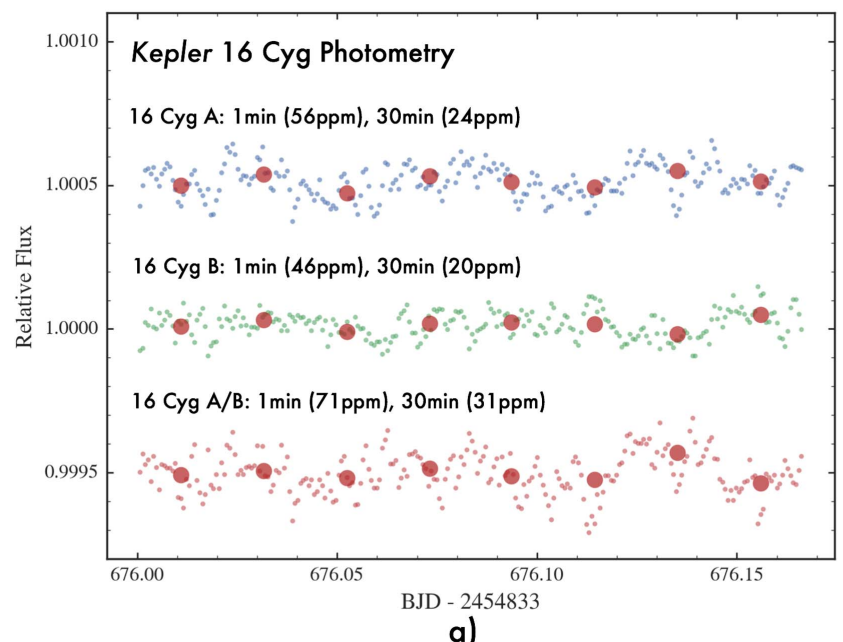

a)

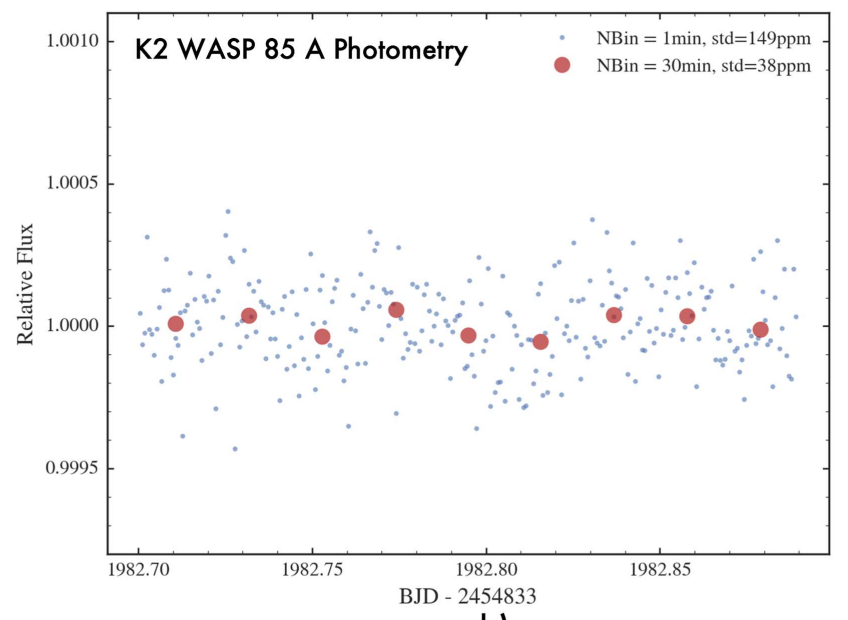

b)

Figure 18. Detrended Kepler and $K 2$ short-cadence photometry of the 16 Cyg system (a) and WASP- 85 A (b). The data are plotted on the same scale. (a) 4 hr of Kepler short-cadence data of 16 Cyg A used in Figure 14. Also shown is the same period for 16 Cyg B, along with the Kepler differential light curve for 16 Cyg A using 16 Cyg B as a reference star (light curve of 16 Cyg A divided by the light curve of 16 Cyg B). (b) K2 data for WASP- 85 A used in Figure 15 . These data were retrieved from MAST and the Everest 2.0 Web site, for panels (a) and (b), respectively.

correlated noise is often not clear without the light curve in hand; therefore, to estimate these values, we did either of the

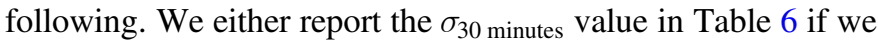
can specifically read the value from the paper (e.g., from a precision versus bin plot), or if the $\sigma_{30 \text { minutes }}$ value was not clearly visible from the paper, we calculated $\sigma_{30 \text { minutes }}$ by performing a best-fit transit fit using the published light-curve data points from the the paper. In the ensuing discussion, we choose to keep the comparison between the optical and NIR photometric precisions separate, due to the dissimilar systematics and instrument technologies involved.

Using the $\sigma_{1 \text { minutes }}$ metric in the optical, we see that the 16 Cygni observations presented here with $\sigma_{1 \text { minutes }}=300 \mathrm{ppm}$ have the overall highest precision, with other efforts coming very close. Notably, these include Kundurthy et al. (2013), achieving a precision of $\sigma_{1 \text { minutes }}=306 \mathrm{ppm}$ through telescope defocusing also on the $3.5 \mathrm{~m}$ telescope at APO. We discuss the Kundurthy et al. (2013) observations further below as a comparison between defocused and diffuser-assisted observations.

Using the $\sigma_{30 \text { minutes }}$ metric, we see that diffuser-assisted precision levels are already matching some of the best published photometric precisions presented in the literature. Notably, these efforts include Colón et al. (2012), achieving $\sigma_{30 \text { minutes }}=\sim 65 \mathrm{ppm}$ using tunable filter narrow-band spectrophotometry on the $10.4 \mathrm{~m}$ GTC telescope,${ }^{27}$ and Johnson et al. (2009), achieving $\sigma_{30 \text { minutes }}=72_{-16}^{+26} \mathrm{ppm}$ precision using the Orthogonal Parallel Transfer Imaging Camera on the $2.2 \mathrm{~m}$ telescope at Maunakea, ${ }^{28}$ and Fukui et al. (2016), achieving $24_{-7}^{14} \mathrm{ppm}$ precision using the MuSCAT imager on the $1.88 \mathrm{~m}$ at the Okayama Astrophysical Observatory in Japan. ${ }^{29}$ For Colón et al. (2012) and Johnson et al. (2009) to achieve photometric precision levels similar to the precisions achieved in this work is not unexpected for a few reasons. First, their

\footnotetext{
27 Value read from the best precision versus bin plot presented in Colón et al. (2012).

${ }^{28}$ Value calculated by fitting a best-fit transit to the published light-curve data from Johnson et al. (2009).

${ }^{29}$ Value calculated by fitting a best-fit transit to the published light-curve data from Fukui et al. (2016).
}

efforts are a specialized implementation of the general techniques that we promote (Colón et al. 2012 using narrowband filters to reduce systematics and spreading out the light over many pixels, and Johnson et al. 2009 deterministically molding the PSF to a broad and stable shape throughout the observations). Taking into account that the scintillation noise is further averaged out with larger telescope diameters as $D^{-2 / 3}$ (see Figure 14) further places these observations at very similar values.

In performing this analysis, we noted that some of the measurements took large excursions down well below the expected Gaussian white noise limit-similar to our TRES-3b observations (formally achieving $54_{-14}^{+23} \mathrm{ppm}$ precision in 30 minute bins, but with an expected photon-limit value of 101 ppm; see Figure 16). This happened notably for Colón et al. (2012), who achieved $\sim 65 \mathrm{ppm}$ precision in 30 minute bins, with a Gaussian expected value of $93 \mathrm{ppm}$, and Fukui et al. (2016), who formally achieved $24_{-7}^{14} \mathrm{ppm}$ precision in 30 minute bins, with a Gaussian expected value of $80 \mathrm{ppm}$. Similar to our discussion above in Section 5.2.2 for our TRES-3b observations, we argue that these values are likely an overestimate of the actual precision due to binninginduced fluctuations at larger bin sizes, and we suggest that the Gaussian expected values are likely a better estimate of the actual achievable precision. We include both values in Table 6 for completeness.

In the NIR, we compare the photometric precision achieved here to two other high-precision NIR photometry efforts in the literature. First, we compare our observations to the defocused observations performed by Croll et al. (2011), who observed the secondary eclipse of WASP 12 in the $J, H$, and $K_{S}$ bands, and second, to the defocused observations performed by Zhao et al. (2014), who observed the secondary eclipse of HAT-P$32 \mathrm{Ab}$ in the $H$ and $K$ bands. To perform a head-to-head comparison with the Croll et al. (2011) and Zhao et al. (2014) results, we specifically compare our photometric precision to their $K_{S}$ observations. Based on the $\sigma_{1}$ minutes metric, Croll et al. (2011) achieve a better precision than our precision in the NIR, but based on the $\sigma_{30 \text { minutes }}$ metric, we see that the precision of our NIR diffuser-assisted observations is better. These early 


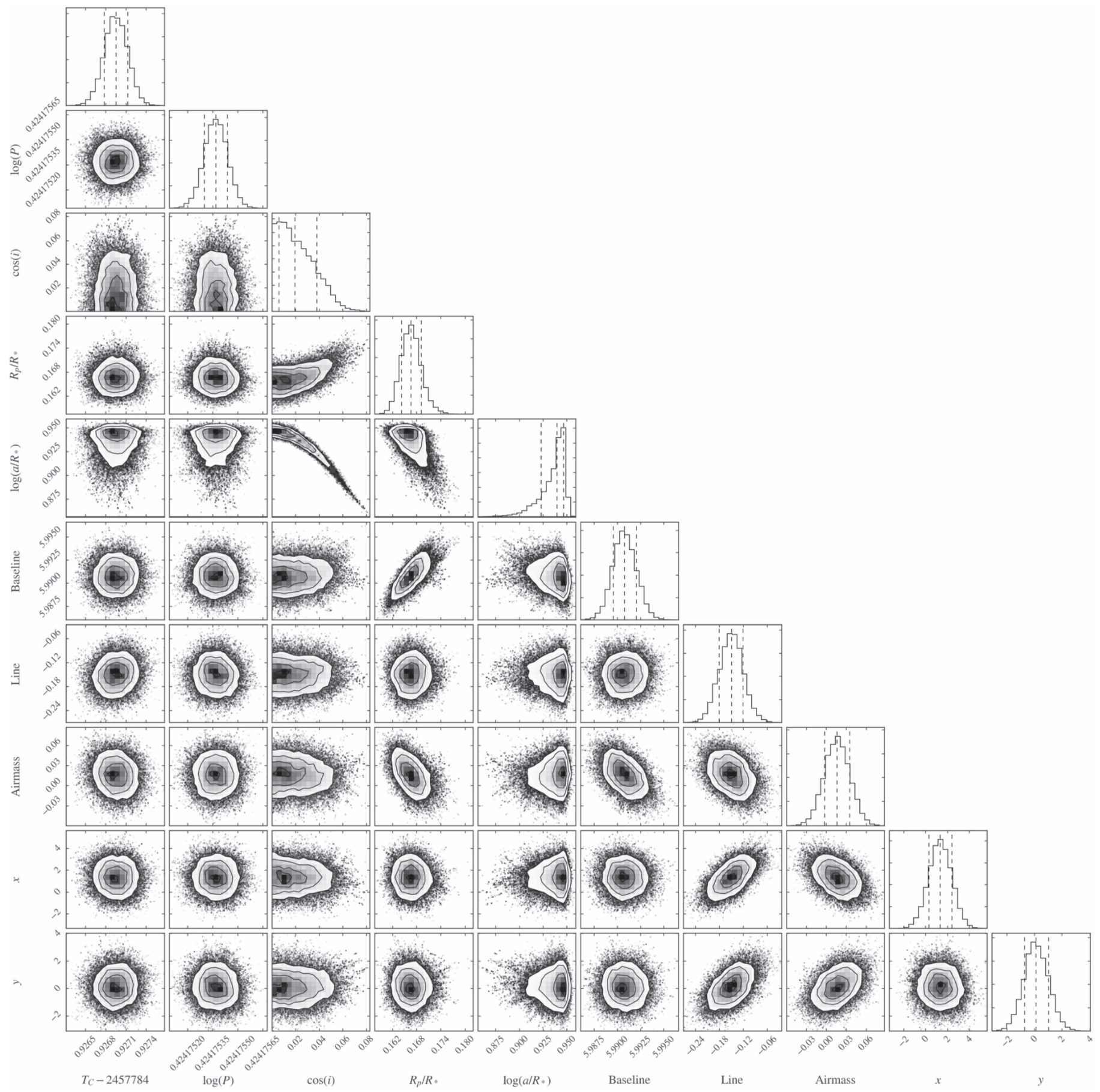

Figure 19. Corner plot from WASP-85 A b data. Plot created using corner.py (Foreman-Mackey 2016).

diffuser-assisted observations with WIRC thus suggest that the diffuser on WIRC is enabling a reliable path for performing routine high-precision observations in the future.

To perform a photometric precision comparison between the diffuser-assisted method and the defocusing method, perhaps the most direct comparison between the two is between our 16 Cyg A photometry and the work by Kundurthy et al. (2013), who performed defocused observations using the AGILE instrument (Mukadam et al. 2011), also on the $3.5 \mathrm{~m} \mathrm{ARC}$ telescope at APO. Such a comparison normalizes the telescope size and observing site out of the equation, but there are still differences in the target observed and the instrument setups (e.g., AGILE allowed for $100 \%$ efficiency). In the NIR, we can make a similar comparison between the work presented here and the work by Zhao et al. (2014), performing defocused observations also using the WIRC instrument on the 200 inch Hale telescope at Palomar. Acknowledging uncertainties in the different observing conditions and other technical factors, overall, Table 6 suggests that diffuser-assisted observations can match or exceed defocused observations with specialized instruments. The main benefit with defocused observations is that the size of the PSF FWHM can be tuned to the observing conditions, but with the downside of being susceptible to PSF variations throughout the night. However, with diffusers and 


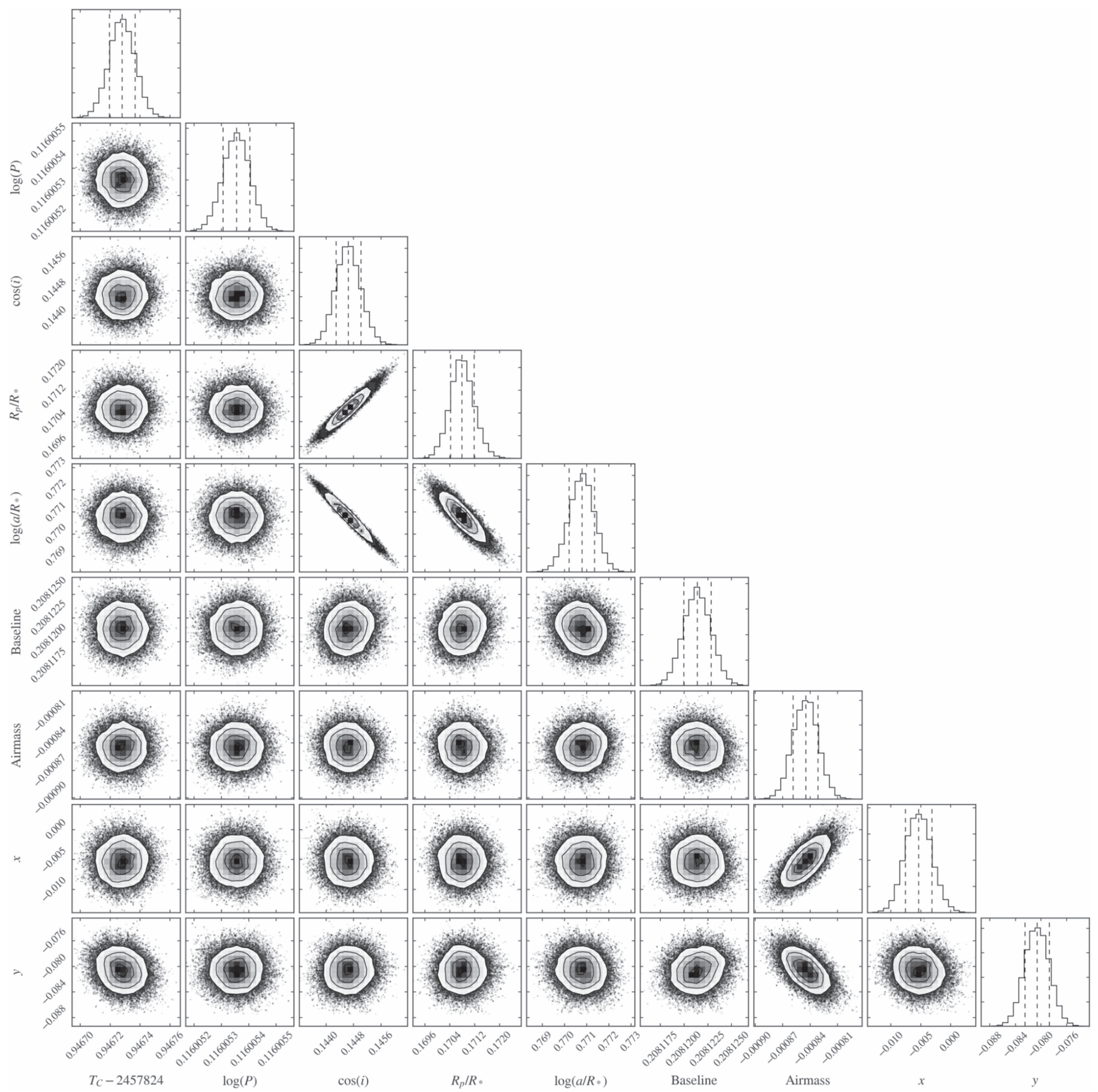

Figure 20. Corner plot from TRES-3b data. Plot created using corner. py (Foreman-Mackey 2016).

their robust PSF stabilization capabilities, we argue that we can more consistently reach precision levels such as those reported in Table 6, even in less than optimal observing conditions (such as our 16 Cyg observations), assuming the availability of a good reference star.

Of interest for future TESS follow-up, we note that that our demonstrated precision in the optical with ARCTIC in 30 minutes matches or surpasses the expected precision of TESS across the different magnitudes observed. For bright stars $\left(I_{C}<7\right)$, TESS is expected to be limited by systematic noise sources at the $\sim 60 \mathrm{ppm}$ level in $1 \mathrm{hr}$ (Sullivan et al. 2015). Assuming a white noise scaling, this corresponds to $\sim 84 \mathrm{ppm}$ in
30 minutes. Our observations of $16 \mathrm{Cyg} \mathrm{A}$, demonstrating $62_{-16}^{+26} \mathrm{ppm}$ in 30 minutes (Figure 14 ) on an $I=5.1$ star, are thus at a similar precision level. Second, as discussed by Sullivan et al. (2015), the expected precision of TESS around an $I_{C}=10$ mag star - a brightness similar to that of WASP-85 A and TRES-3b-is $\sim 200 \mathrm{ppm}$ in $1 \mathrm{hr}$. Likewise, assuming a white noise scaling, this precision corresponds to $200 \sqrt{2}=$ $\sim 282 \mathrm{ppm}$ in 30 minutes. With our WASP-85 A b and TRES$3 \mathrm{~b}$ transit observations, we demonstrate a 30 minute precision better than this by factors of 1.6 and 2.8 , respectively. This demonstrates that ARCTIC with a diffuser will be capable of following up TESS targets with TESS-like precision across a 
Table 5

Median Values and 68\% Confidence Intervals for the Transit Fit Parameters for WASP-85 A b and TRES-3b

\begin{tabular}{|c|c|c|c|}
\hline Parameter & Description & WASP-85 A b & TRES-3b \\
\hline$\overline{T_{C}\left(\mathrm{BJD}_{\mathrm{TDB}}\right)}$ & Transit midpoint & $2457784.92695_{-0.00017}^{+0.00017}$ & $2457824.946728_{-0.000008}^{+0.000009}$ \\
\hline$P$ (days) & Orbital period & $2.6556777_{-0.0000004}^{+0.000004}$ & $1.3061870_{-0.0000001}^{+0.0000001}$ \\
\hline$R_{p} / R_{*}$ & Radius ratio & $0.1666_{-0.0024}^{+0.0025}$ & $0.17079_{-0.00037}^{+0.00039}$ \\
\hline$R_{p}\left(R_{\oplus}\right)$ & Planet radius & $16.99_{-0.48}^{+0.50}$ & $15.44_{-0.41}^{+0.41}$ \\
\hline$R_{p}\left(R_{J}\right)$ & Planet radius & $1.515_{-0.043}^{+0.044}$ & $1.377_{-0.036}^{+0.037}$ \\
\hline$\delta$ & Transit depth & $0.02775_{-0.00080}^{+0.00085}$ & $0.02917_{-0.00013}^{+0.00013}$ \\
\hline$a / R_{*}$ & Normalized orbital radius & $8.71_{-0.33}^{+0.14}$ & $5.8991_{-0.0079}^{+0.0077}$ \\
\hline$a(\mathrm{AU})$ & Semimajor axis & $0.0376_{-0.0015}^{+0.0012}$ & $0.02273_{-0.00060}^{+0.00060}$ \\
\hline$i\left(^{\circ}\right)$ & Transit inclination & $88.89_{-1.10}^{+0.77}$ & $81.683_{-0.021}^{+0.021}$ \\
\hline$b$ & Impact parameter & $0.17_{-0.12}^{+0.15}$ & $0.8533_{-0.0010}^{+0.0011}$ \\
\hline$e$ & Eccentricity & 0 (adopted) & 0 (adopted) \\
\hline$\omega\left(^{\circ}\right)$ & Argument of periastron & 0 (adopted) & 0 (adopted) \\
\hline$T_{\mathrm{eq}}(\mathrm{K})$ & Equilibrium temperature & $1366_{-21}^{+28}$ & $1645_{-22}^{+22}$ \\
\hline$T_{14}$ (days) & Transit duration & $0.1124_{-0.0011}^{+0.0016}$ & $0.05728_{-0.00004}^{+0.00004}$ \\
\hline$\tau$ (days) & Ingress/egress duration & $0.0165_{-0.0006}^{+0.0016}$ & $0.02864_{-0.00002}^{+0.00002}$ \\
\hline$T_{S}\left(\mathrm{BJD}_{\mathrm{TDB}}\right)$ & Time of secondary eclipse & $2457786.25479_{-0.00017}^{+0.00017}$ & $2457825.5998215_{-0.000008}^{+0.000009}$ \\
\hline
\end{tabular}

large range of magnitudes, assuming the availability of good reference stars. We similarly expect that the WIRC instrument with a diffuser will be a workhorse instrument in following up with TESS targets at high precision. However, with WIRC operating in the NIR, the comparison with the expected TESS precision levels - which are in the optical-is not strictly analogous to comparing to our ARCTIC optical precision levels to TESS, as, e.g., the photon count levels for $K_{S}=10$ mag stars are different from those of $I_{C}=10$ mag stars.

To reach photometric precision levels similar to those reported in Table 6 requires a judicious attention to minimizing all sources of photometric noise. For the brightest stars (see Figure 2), scintillation noise is larger than the photon noise across different telescope systems on the ground. The impact of scintillation can be minimized by increasing the exposure time and maximizing the duty cycle, and through observing with larger telescopes at higher altitudes. Insofar as to allow for increased exposure times, diffusers do not specifically minimize scintillation noise. There have been suggestions in the literature on how to further minimize scintillation effects (Dravins et al. 1998; Osborn et al. 2011), including, in particular, the conjugate plane photometry technique, which consists of putting a mask in the optical train at the conjugate plane of the scintillation layer to block out unwanted rays from the scintillation layer in the upper atmosphere. In doing so, the scintillation layer is effectively moved to the ground layers of the atmosphere, where the coherence angle is larger (on the order of $0^{\circ} .5$ ), allowing the intensity variations of the target star to be corrected by a comparison star. Although it is a promising method to suppress scintillation errors, and thus the photometric error budget as a whole, conjugate plane photometry requires a specific optical setup and specialized instrumentation. Furthermore, it also benefits from simultaneous SCIDAR measurements of the atmospheric turbulence to measure where the turbulent scintillation layer is and thus to inform where the conjugate mask should be placed (Osborn et al. 2011). However, in our efforts to achieve precision photometry from the ground, we urge the community to consider such ways to suppress scintillation errors, and incorporating a diffuser with a scintillation-suppressing instrument would potentially open a path to achieving even better precisions than those presented here.

\subsection{Adaptability of Diffusers in other Systems}

The most straightforward way to incorporate a diffuser in a telescope imaging system is in a standard filter wheel. This is most efficient if the telescope has two filter wheels, placing the diffuser in one slot of one filter wheel and retaining the capability to select a filter in the other wheel. If only one filter wheel is available, a diffuser could be combined with a filter in a single filter wheel slot by placing them back-to-back in the slot. This configuration could potentially cause back-reflections and ghosting, which could be corrected by adding a small wedge between the filter and a diffuser. A more permanent solution would be imprinting a diffuser pattern directly onto the filter. Although we have not developed such a device, it is an interesting avenue for further study.

We studied the PSFs of off-the-shelf top-hat diffusers. The off-the-shelf diffusers studied are capable of spreading out the light deterministically over a large number of pixels, which can easily be calculated using Equation (8), or through precisely modeling diffusers using Zemax OpticStudio. Although all of the off-the-shelf diffusers tested produce an approximate a tophat-shaped PSF, we observed them to have a speckle pattern of $\sim 20 \%-40 \%$ of the total intensity along with having less than optimal wings. Although the speckle pattern is observed to be completely stable in the lab and on sky, we demonstrate that both the speckle pattern and the wing fall-off can be further reduced through optimizing the design of the diffuser for a given application. This optimization process improves the signal within the aperture, but we demonstrate that using offthe-shelf diffusers can yield very high-precision photometry even on small telescopes.

Rotation is effective at removing the speckles observed on the diffuser PSF. Although we found that even without rotation the diffuser PSF is extremely stable, rotation helps further smooth out the PSF. Rotational smoothing effectively increases the dynamic range of the observations, removing any spikes or speckles that might potentially saturate the detector.

Diffusers, however, are not free of limitations. Their main drawback is their fixed PSF size. In crowded fields, such as 


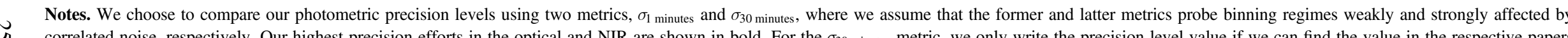

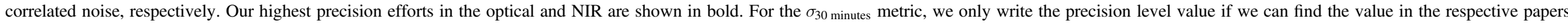

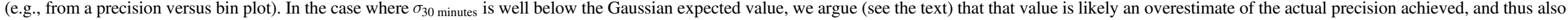
write the Gaussian expected value (marked with a (f)). Overall, diffuser-assisted photometry, both in the optical and the NIR, is achieving some of the best photometry in the literature.

${ }^{a}$ Using the $4 \mathrm{~m}$ telescope at Kitt Peak National Observatory, using a Corning glass filter with a central wavelength of $472 \mathrm{~nm}$ and an FWHM of $166.5 \mathrm{~nm}$, observing stars in the M67 cluster.

${ }^{\mathrm{b}}$ Calculated from the published light-curve data points, calculating residuals versus binning through fitting a best-fit transit model (in a similar fashion to the diffuser-assisted observations).

${ }^{c}$ Using the Orthogonal Parallel Transfer Imaging Camera on the $2.2 \mathrm{~m}$ University of Hawaii telescope at Maunakea, observing the transit of WASP $10 \mathrm{~b}$.

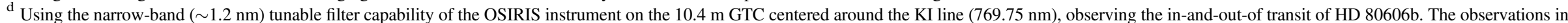

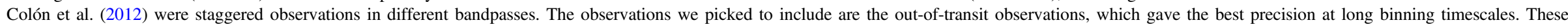

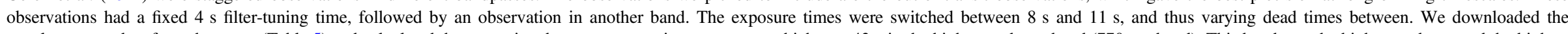

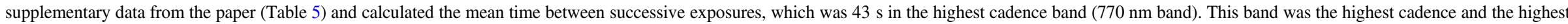
precision at the longest bin sizes.

${ }^{\mathrm{e}}$ Estimated from precision versus binning plots in the respective papers.

f The values are the Gaussian expected precision level in 30 minutes and are likely closer to the truer precision achieved in these cases.

${ }^{\mathrm{g}}$ Using AGILE, a charge transfer CCD enabling 100\% duty cycle (Kundurthy et al. 2011; Mukadam et al. 2011), on the $3.5 \mathrm{~m}$ telescope at Apache Point Observatory, observing the transit of XO-2b.

${ }^{\mathrm{h}}$ Using the $3.58 \mathrm{~m}$ ESO New Technology Telescope at La Silla, Chile, using the Gunn $r$ filter (ESO Filter \#784) to observe the transit of WASP $50 \mathrm{~b}$.

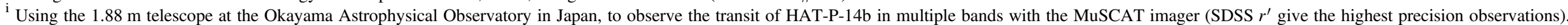

${ }^{\mathrm{j}}$ Using the $3.6 \mathrm{~m}$ Canada-France-Hawaii Telescope (CFHT) at Maunakea to observe the secondary eclipse of WASP $12 \mathrm{~b}$.

${ }^{\mathrm{k}}$ Using the $5.0 \mathrm{~m}$ Hale 200 inch telescope at Palomar to observe the secondary eclipse of HAT-P-32Ab. 
fields toward the center of the galaxy, sources can overlap. This can similarly be an issue for closely separated binary stars, but at least in the case of our WASP-85 A b observations, we were able to demonstrate the capability to maintain a high level of precision throughout the observations, even with completely overlapping PSFs. Another limitation is background sky-noise, which starts to dominate for stellar PSFs spread out over many pixels, an especially important consideration for faint stars and in the NIR. In practice, for our diffuser-assisted observations with ARCTIC, we start to see significant effects from background sky noise at around SDSS $i^{\prime}$ magnitudes of $\sim 13-14$. One way to solve this problem and enable adjustability in the PSF size involves varying the distance of the diffuser from the detector to deterministically optimize the size of the PSF for a given observation. This, however, would require a dedicated pistoning mechanism that could move the diffuser toward and away from the detector, which might not always be possible. Another option would be to have a separate filter wheel with diffusers of different opening angles. The price of such a setup could be kept to a minimum by using offthe-shelf polymer diffusers costing $\sim \$ 250$ each.

An additional second-order effect that we have observed with our final optical diffuser on ARCTIC is that around very bright stars, the diffuser forms images of two equally bright spots equidistant from the main PSF $\sim 800$ pixels away from the central PSF that have intensities proportional to the net counts in the central PSF. We note that these spots are faint: the total counts observed in the diffractive orders is $\sim 10^{4}$ counts for $4 \times 10^{7}$ counts in the central PSF. We speculate that the spots observed are the +1 and -1 order of the diffuser acting as a diffraction grating, as we observe that the exact separation of the spots is wavelength dependent. Through modeling the diffuser in the telescope as a transmissive diffraction grating in Zemax, we can accurately replicate the spot locations, assuming a groove spacing of $6.0 \mu \mathrm{m}$. We attribute this grating effect to potential periodic systematics in the laser writer during the diffuser fabrication process, where $6 \mu \mathrm{m}$ might correspond to the row step size or an integer multiple of the step size. We stress that the effect has little-to-no effect on the photometric precision, as it is a static effect. If, however, the spot was to completely overlap one of the reference stars, it would introduce an additional source of systematic error, correlated with the signal being studied. This can be easily solved by rotating the diffuser or by rotating the focal plane array with respect to the diffuser to ensure that the ghost spots from one star do not coincide with a target or a reference star in the field.

\subsection{Using Diffusers in Space?}

Engineered Diffusers ${ }^{\mathrm{TM}}$ are available with direct etching of the diffusing surface on fused silica, making it suitable for space applications due to its radiation-resistant properties. As such, Engineered Diffusers ${ }^{\mathrm{TM}}$ have been considered in the lab for space use for the CHEOPS mission by Magrin et al. (2014). Their aim was to shape the PSF into nearly a top-hat with $30^{\prime \prime}$ FWHM, corresponding to a 30 pixel FWHM due to the CHEOPS plate scale of 1 !! 0 pixel $^{-1}$.

Magrin et al. (2014) studied in the lab the PSF-shaping capabilities of an off-the-shelf Engineered Diffuser ${ }^{\mathrm{TM}}$ (part number: EDC-0.25-A-1r) with an opening angle of $0^{\circ} 25$. This is the same 0.25 polymer diffuser pattern as we studied (see Table 2), replicated on a $1^{\prime \prime}$ circular substrate instead of the $2^{\prime \prime} \times 2^{\prime \prime}$ square substrate diffuser we studied. Magrin et al. (2014) also studied shaping the PSF to the desired top-hat form factor using a microlens array from RPC Photonics. In their study, Magrin et al. (2014) found that the resulting diffuser PSF shape did not approximate a top-hat shape, but rather had a broad Gaussian-like output with numerous speckles. This is similar to our results with the 0.25 diffuser, as we show in Figure 9: the 0.25 diffuser indeed gave the largest amplitude speckles of the off-the-shelf diffusers tested. However, in this paper, we demonstrated that the speckling is completely stable throughout the observations, and that both the speckling and the fall-off of the wings can be further optimized to deliver a homogeneous PSF shape approximating a top-hat shape. The CHEOPS team decided to not fly with a diffuser, choosing to defocus the telescope instead, due to the spikiness of the observed diffused PSF and the additional risk associated with what to them was unproven technology for high-precision photometry applications.

We now consider diffusers a proven technology in achieving high precision from the ground. We believe that in space too, diffusers will be beneficial for high photometric precision surveys of bright nearby targets - especially with a diffuser customized for the telescope system. Diffusers minimize flatfielding errors and jitter effects in the pointing of the telescope, a major source of systematics in space telescope systems. We speculate that diffusers could enable small spacecraft, such as cubesats, with less than optimal pointing precision to achieve high-precision photometry. This could be especially beneficial, e.g., to perform long-term uninterrupted photometric monitoring of RV planet hosts to separate stellar activity from exoplanet signals.

\subsection{Future Outlook}

Our results highlight the potential of using ground-based diffuser-assisted photometry to perform routine high-precision follow-up observations of nearby bright-planet systems, such as those that are being detected with $K 2$ and those that will be detected by the TESS mission in the future. Diffuser-assisted photometry systems from the ground will allow for consistent rapid reconnaissance follow-up observations for TESS targets, and by spreading out diffusers to other telescopes, we expect that telescopes equipped with diffusers will serve as workhorse instruments in following up TESS candidates in the future. Through this effort, we can secure an up-to-date target list of precisely characterized planets for further study of exoplanet atmospheres with facilities such as Hubble, JWST, and future $30 \mathrm{~m}$ class telescopes.

In the interest of spreading this effort and technology to the community for use on other telescopes, our intent is to make our optimized diffuser pattern for the optical diffuser available to the community through RPC Photonics.

\section{Summary}

We describe a reliable technique to achieve space-quality photometric precision on nearby bright stars using groundbased telescopes by coupling a beam-shaping diffuser capable of molding a varying stellar input to a broad stabilized top-hat shape without defocusing the telescope. Spreading the light over many pixels minimizes flat-field errors and telescopeguiding errors, allowing exposure times to be increased to effectively gather more photons while averaging over 
scintillation errors. Using this technology, we demonstrated some of the highest photometric precisions from the ground on the ARCTIC camera on the Apache Point $3.5 \mathrm{~m}$ telescope in the optical and the WIRC camera at the 200 inch Hale telescope at Palomar in the NIR. Specifically, on ARCTIC, we achieve $62_{-16}^{+26} \mathrm{ppm}$ precision in 30 minute bins on a nearby bright star 16 Cyg A, and with WIRC we achieve $137_{-36}^{+64} \mathrm{ppm}$ precision in an early test demonstration of diffusers in the NIR. Additionally, we demonstrate that diffuser-assisted observations on small-type telescopes are also capable of delivering precision photometry ( $\sim 300 \mathrm{ppm}$ in 30 minutes) in observations of 55 Cnc using the Penn State PlaneWave CDK 24 inch telescope.

In this paper, we discussed how diffusers operate, reporting our lab and on-sky tests with different telescope systems, demonstrating that diffusers offer broadband compatibility in both the optical and NIR. Moreover, through both numerical simulations and on-sky efforts, we demonstrate that diffusers work in both collimated and converging telescope beams. Being relatively simple and inexpensive devices, diffusers can be easily incorporated into a variety of telescope systems to improve their photometric precision on nearby bright stars. The true power of this technique is making high photometric precision levels widely accessible without specialized instrumentation or the rarest observing conditions. In light of the upcoming TESS mission, which will require precise and timely follow-up from the ground to validate and characterize exciting transiting targets, our intent is to work with the community to spread out diffuser technology by offering our optimized diffuser pattern through RPC Photonics.

We thank the anonymous referee for a thoughtful reading of the manuscript, and for useful suggestions and comments. We gratefully acknowledge the work and assistance of Tasso Sales and Laura Weller-Brophy at RPC Photonics, without whose help this project would not have been possible. G.K.S. wishes to thank Eric Ford for helpful discussions on MCMC fitting and inference. This work was directly seeded and supported by a Scialog grant from the Research Corporation for Science Advancement (Rescorp) to S.M., L.H., and J.W. This work was partially supported by funding from the Center for Exoplanets and Habitable Worlds. The Center for Exoplanets and Habitable Worlds is supported by the Pennsylvania State University, the Eberly College of Science, and the Pennsylvania Space Grant Consortium. G.K.S. acknowledges support from the Leifur Eiriksson Foundation Scholarship. This work was supported by NASA Headquarters under the NASA Earth and Space Science Fellowship Program Grant NNX16AO28H. This work was performed in part under contract with the Jet Propulsion Laboratory (JPL) funded by NASA through the Sagan Fellowship Program executed by the NASA Exoplanet Science Institute. We acknowledge support from NSF grants AST-1006676, AST-1126413, AST-1310885, and AST1517592, the NASA Astrobiology Institute (NAI; NNA09DA76A), and PSARC.

These results are based on observations obtained with the Apache Point Observatory $3.5 \mathrm{~m}$ telescope which is owned and operated by the Astrophysical Research Consortium, the Hale 200 inch Telescope at Palomar Observatory, and the Planewave CDK 24 Telescope operated by the Penn State Department of Astronomy \& Astrophysics at Davey Lab Observatory. The Palomar Hale 200 inch telescope is operated by Caltech and the Jet Propulsion Laboratory. This paper includes data collected by the Kepler telescope. The Kepler and $K 2$ data presented in this paper were obtained from the Mikulski Archive for Space Telescopes (MAST). The Space Telescope Science Institute is operated by the Association of Universities for Research in Astronomy, Inc., under NASA contract NAS5-26555. Support for MAST for non-HST data is provided by the NASA Office of Space Science via grant NNX09AF08G and by other grants and contracts. Funding for the $K 2$ Mission is provided by the NASA Science Mission directorate. This research made use of the NASA Exoplanet Archive, which is operated by the California Institute of Technology, under contract with the National Aeronautics and Space Administration under the Exoplanet Exploration Program.

Facilities: ARC, Hale, DAVEY:0.6m, Kepler.

Software: AstroImageJ (Collins et al. 2017), astropy (Astropy Collaboration et al. 2013), astroscrappy, BATMAN (Kreidberg 2015), corner . py (Foreman-Mackey 2016), emcee (Foreman-Mackey et al. 2013), Everest 2.0 (Luger et al. 2017), EXOFAST (Eastman et al. 2013), MC3 (Cubillos et al. 2017), TERRASPEC (Bender et al. 2012), Zemax OpticStudio.

\section{Appendix A Kepler and $K 2$ Photometry}

Figure 18(a) shows the section of $4 \mathrm{hr}$ short-cadence photometry of $16 \mathrm{Cyg}$ A (blue curve, unbinned) and $16 \mathrm{Cyg}$ B (green curve, unbinned), as observed by Kepler in Quarter 7, used in Figure 14. Additionally shown in Figure 18(a) is the differential photometry of $16 \mathrm{Cyg} \mathrm{A}$, using $16 \mathrm{Cyg} \mathrm{B}$ as a reference star (red curve, unbinned, denoted $16 \mathrm{Cyg} \mathrm{A} / \mathrm{B}$ ). Furthermore, we also show the 30 minute binned points for each curve (thick red dots), along with the unbinned and binned precisions for this segment for the three light curves. We chose this specific time window to be representative of the Kepler data of 16 Cyg A, as in 30 minute bins the rms scatter of this $4 \mathrm{hr}$ segment matches well with the 30 minute CDPP precision across the whole Quarter 7 of this star. These data were easily retrievable from MAST and detrended as described in the text.

Similarly, Figure 18(b) shows the section of $4.5 \mathrm{hr}$ shortcadence photometry of WASP-85 A, as observed by $K 2$ in Campaign 1, used in Figure 15. The short-cadence detrended $K 2$ data of WASP-85 A was retrieved and detrended using the Everest $2.0 \mathrm{Web}$ site and pipeline, ${ }^{30}$ but the data are also readily available from MAST.

We note that we can visibly see evidence of correlated noise structure in both the 16 Cyg and the WASP-85 A photometry, which we attribute to astrophysical activity. The scatter for WASP-85 A is visibly larger than that for the 16 Cyg system due to its faintness.

\section{Appendix B Transit-fitting Posterior Plots}

\section{B.1. Transit of WASP-85 A $b$}

The two-dimensional corner plot of the resulting chains for WASP-85 A b is shown in Figure 19.

\footnotetext{
${ }^{30}$ Everest 2.0 Web site: http://staff.washington.edu/rodluger/everest/ catalog.html.
} 


\section{B.2. Transit of TRES-3b}

The two-dimensional corner plot of the resulting chains for TRES-3b is shown in Figure 20.

\section{ORCID iDs}

Gudmundur Stefansson (1) https://orcid.org/0000-00017409-5688

Suvrath Mahadevan (i) https://orcid.org/0000-00019596-7983

Leslie Hebb (10) https://orcid.org/0000-0003-1263-8637

John Wisniewski (i) https://orcid.org/0000-0001-9209-1808

Brett Morris (1) https://orcid.org/0000-0003-2528-3409

Sam Halverson (i) https://orcid.org/0000-0003-1312-9391

Ming Zhao (i) https://orcid.org/0000-0002-4258-9517

Jason Wright (i) https://orcid.org/0000-0001-6160-5888

Suzanne Hawley (1) https://orcid.org/0000-0002-6629-4182

Lea M. Z. Hagen (1) https://orcid.org/0000-0001-8918-1597

Thomas Beatty (i) https://orcid.org/0000-0002-9539-4203

Chad Bender (1) https://orcid.org/0000-0003-4384-7220

Paul Robertson (1) https://orcid.org/0000-0003-0149-9678

\section{References}

Astropy Collaboration, Robitaille, T. P., Tollerud, E. J., et al. 2013, A\&A, 558, A33

Bastien, F. A., Stassun, K. G., Pepper, J., et al. 2014, AJ, 147, 29

Batalha, N. E., Kempton, E. M.-R., \& Mbarek, R. 2017, ApJL, 836, L5

Bender, C. F., Mahadevan, S., Deshpande, R., et al. 2012, ApJL, 751, L31

Benneke, B., Werner, M., Petigura, E., et al. 2017, ApJ, 834, 187

Bessell, M. S. 1990, PASP, 102, 1181

Blecic, J., Harrington, J., Madhusudhan, N., et al. 2013, ApJ, 779, 5

Borucki, W. J., Koch, D., Basri, G., et al. 2010, Sci, 327, 977

Brown, D. J. A., Anderson, D. R., Armstrong, D. J., et al. 2014, arXiv:1412.7761

Burke, C. J., Christiansen, J. L., Mullally, F., et al. 2015, ApJ, 809, 8

Claret, A., \& Bloemen, S. 2011, A\&A, 529, A75

Collins, K. A., Kielkopf, J. F., Stassun, K. G., \& Hessman, F. V. 2017, arXiv: 1701.04817

Colón, K. D., Ford, E. B., Redfield, S., et al. 2012, MNRAS, 419, 2233

Cowan, N. B., Greene, T., Angerhausen, D., et al. 2015, PASP, 127, 311

Croll, B., Lafreniere, D., Albert, L., et al. 2011, AJ, 141, 30

Crossfield, I. J. M., Petigura, E., Schlieder, J. E., et al. 2015, ApJ, 804, 10

Cubillos, P., Harrington, J., Loredo, T. J., et al. 2017, AJ, 153, 3

Cubillos, P., Harrington, J., Madhusudhan, N., et al. 2013, ApJ, 768, 42

Davies, G. R., Chaplin, W. J., Farr, W. M., et al. 2015, MNRAS, 446, 2959

Dawson, R. I., \& Fabrycky, D. C. 2010, ApJ, 722, 937

de Ugarte Postigo, A., Roming, P., Thone, C. C., et al. 2016, Proc. SPIE, 9908, 990840

Dravins, D., Lindegren, L., Mezey, E., \& Young, A. T. 1998, PASP, 110, 610

Eastman, J., Gaudi, B. S., \& Agol, E. 2013, PASP, 125, 83

Ford, E. B. 2006, ApJ, 642, 505

Foreman-Mackey, D. 2016, JOSS, 24

Foreman-Mackey, D., Hogg, D. W., Lang, D., \& Goodman, J. 2013, PASP, 125,306
Fukugita, M., Ichikawa, T., Gunn, J. E., et al. 1996, AJ, 111, 1748

Fukui, A., Narita, N., Kawashima, Y., et al. 2016, ApJ, 819, 27

Gilliland, R. L., Brown, T. M., Kjeldsen, H., et al. 1993, AJ, 106, 2441

Gilliland, R. L., Chaplin, W. J., Dunham, E. W., et al. 2011, ApJS, 197, 6

Gilliland, R. L., Jenkins, J. M., Borucki, W. J., et al. 2010, ApJL, 713, L160

Howell, S. B., Everett, M. E., Tonry, J. L., Pickles, A., \& Dain, C. 2003, PASP, 115,1340

Huehnerhoff, J., Ketzeback, W., Bradley, A., et al. 2016, Proc. SPIE, 9908, 99085H

Jiang, I.-G., Yeh, L.-C., Thakur, P., et al. 2013, AJ, 145, 68

Johnson, J. A., Winn, J. N., Cabrera, N. E., \& Carter, J. A. 2009, ApJL, 692, L100

Knutson, H. A., Fulton, B. J., Montet, B. T., et al. 2014, ApJ, 785, 126

Kornilov, V. 2012, MNRAS, 425, 1549

Kreidberg, L. 2015, PASP, 127, 1161

Kundurthy, P., Agol, E., Becker, A. C., et al. 2011, ApJ, 731, 123

Kundurthy, P., Barnes, R., Becker, A. C., et al. 2013, ApJ, 770, 36

Lucy, L. B., \& Sweeney, M. A. 1971, AJ, 76, 544

Luger, R., Agol, E., Kruse, E., et al. 2016, AJ, 152, 100

Luger, R., Kruse, E., Foreman-Mackey, D., Agol, E., \& Saunders, N. 2017, ApJ, submitted (arXiv:1702.05488)

Lund, M. N., Kjeldsen, H., Christensen-Dalsgaard, J., Handberg, R., \& Silva Aguirre, V. 2014, ApJ, 782, 2

Magrin, D., Farinato, J., Umbriaco, G., et al. 2014, Proc. SPIE, 9143, 91434L

Mandel, K., \& Agol, E. 2002, ApJL, 580, L171

Mann, A. W., Gaidos, E., \& Aldering, G. 2011, PASP, 123, 1273

McArthur, B. E., Endl, M., Cochran, W. D., et al. 2004, ApJL, 614, L81

Merline, W. J., \& Howell, S. B. 1995, ExA, 6, 163

Metcalfe, T. S., Chaplin, W. J., Appourchaux, T., et al. 2012, ApJL, 748, L10

Morris, G., \& Sales, T. 2006, Structured Screens for Controlled Spreading of Light, US Patent 7.033.736

Močnik, T., Clark, B. J. M., Anderson, D. R., Hellier, C., \& Brown, D. J. A 2016, AJ, 151, 150

Mukadam, A. S., Owen, R., Mannery, E., et al. 2011, PASP, 123, 1423

O’Donovan, F. T., Charbonneau, D., Bakos, G. Á, et al. 2007, ApJL, 663, L37

Osborn, J., Föhring, D., Dhillon, V. S., \& Wilson, R. W. 2015, MNRAS, 452, 1707

Osborn, J., Wilson, R. W., Dhillon, V. S., Avila, R., \& Love, G. D. 2011 MNRAS, 411, 1223

Plavchan, P., Latham, D., Gaudi, S., et al. 2015, arXiv:1503.01770

Potter, D. E. 2006, BAAS, 38, 1173

Ricker, G. R., Winn, J. N., Vanderspek, R., et al. 2014, JATIS, 1, 014003

Sales, T. R., Chakmakjian, S., Morris, G. M., \& Schertler, D. J. 2004, PhoSp, https://www.rpcphotonics.com/pdfs/Light_Tamers.pdf

Southworth, J., Hinse, T. C., Jørgensen, U. G., et al. 2009, MNRAS, 396, 1023

Sozzetti, A., Torres, G., Charbonneau, D., et al. 2009, ApJ, 691, 1145

Sullivan, P. W., Winn, J. N., Berta-Thompson, Z. K., et al. 2015, ApJ, 809, 77

Tonry, J., Burke, B. E., \& Schechter, P. L. 1997, PASP, 109, 1154

Tregloan-Reed, J., \& Southworth, J. 2013, MNRAS, 431, 966

van Dokkum, P. G. 2001, PASP, 113, 1420

Vanderburg, A., Bieryla, A., Duev, D. A., et al. 2016, ApJL, 829, L9

Vanderburg, A., \& Johnson, J. A. 2014, PASP, 126, 948

Villanueva, S., Jr., Eastman, J. D., \& Gaudi, B. S. 2016, ApJ, 820, 87

Wilson, J. C., Eikenberry, S. S., Henderson, C. P., et al. 2003, Proc. SPIE, 4841, 451

Winn, J. N., Matthews, J. M., Dawson, R. I., et al. 2011, ApJL, 737, L18

Young, A. T. 1967, AJ, 72, 747

Zhao, M., Milburn, J., Barman, T., et al. 2012, ApJL, 748, L8

Zhao, M., O’Rourke, J. G., Wright, J. T., et al. 2014, ApJ, 796, 115 